\title{
Accumbal Neurons that are Activated during Cocaine Self-Administration are Spared from Inhibitory Effects of Repeated Cocaine Self-Administration
}

\author{
Laura L Peoples*, 1,2, Alexxai V Kravitz ${ }^{2}$, Kevin G Lynch ${ }^{3}$ and Daniel J Cavanaugh ${ }^{4}$ \\ 'Department of Psychology, University of Pennsylvania, Philadelphia, PA, USA; ${ }^{2}$ Neuroscience Graduate Group, University of Pennsylvania Medical \\ School, Philadelphia, PA, USA; ${ }^{3}$ Department of Psychiatry, University of Pennsylvania Medical School, Philadelphia, PA, USA; ${ }^{4}$ Department of \\ Physiology, University of California, San Francisco, CA, USA
}

\begin{abstract}
Hypoactivity of the accumbens is induced by repeated cocaine exposure and is hypothesized to play a role in cocaine addiction. However, it is difficult to understand how a general hypoactivity of the accumbens, which facilitates multiple types of motivated behaviors, could contribute to the selective increase in drug-directed behavior that defines addiction. Electrophysiological recordings, made during sessions in which rats self-administer cocaine, show that most accumbal neurons that encode events related to drugdirected behavior achieve and maintain higher firing rates during the period of cocaine exposure (Task-Activated neurons) than do other accumbal neurons (Task-Non-Activated neurons). We have hypothesized that this difference in activity makes the neurons that facilitate drug-directed behavior less susceptible than other neurons to the chronic inhibitory effects of cocaine. A sparing of neurons that facilitate drug-directed behavior from chronic hypoactivity might lead to a relative increase in the transmission of neuronal signals that facilitate drug-directed behavior through accumbal circuits and thereby contribute to changes in behavior that characterize addiction (ie differential inhibition hypothesis). A prediction of the hypothesis is that neurons that are activated in relation to task events during cocaine self-administration sessions will show less of a decrease in firing across repeated self-administration sessions than will other neurons. To test this prediction, rats were exposed to 30 daily $(6 \mathrm{~h} /$ day $)$ cocaine self-administration sessions. Chronic extracellular recordings of single accumbal neurons were made during the second to third session and the 30th session. Between-session comparisons showed that decreases in firing were exhibited by Task-Non-Activated, but not by Task-Activated, neurons. During the day 30 session, the magnitude of the difference in firing rate between the two groups of neurons was positively related to the propensity of animals to seek and take cocaine. The findings of the present study are consistent with a basic prediction of the differential inhibition hypothesis and may be relevant to understanding cocaine addiction.

Neuropsychopharmacology (2007) 32, I |4 I- I 158. doi:I0.1038/sj.npp. I30 I203; published online 4 October 2006
\end{abstract}

Keywords: accumbens; addiction; neuroadaptations; dopamine; glutamate; plasticity

\section{INTRODUCTION}

Acute electrophysiological recording studies have consistently demonstrated that a history of repeated exposure to cocaine leads to hypoactivity of accumbal neurons (White et al, 1995a, b; Zhang et al, 1998, 2002; Thomas et al, 2001; Beurrier and Malenka, 2002). Imaging studies in both cocaine-exposed laboratory animals (Porrino et al, 2002; Kaufman et al, 2003; Macey et al, 2004; Febo et al, 2005) and human cocaine addicts (Volkow et al, 1993; London et al,

*Correspondence: Dr LL Peoples, Department of Psychology, University of Pennsylvania, 3720 Walnut Street, Philadelphia, PA 19106, USA, Tel: + I 215898 4253, Fax: + I 2158987301 ,

E-mail: Ipeoples@psych.upenn.edu

Received 17 March 2006; revised 6 July 2006; accepted 27 July 2006 Online publication: 4 August 2006 at http://www.acnp.org/citations/ Npp080406060 173/default.pdf
1999; Volkow and Fowler, 2000) have also documented the presence of hypoactivity within the ventral striatum, and the larger cortico-striato-thalamo-cortical circuit. Based on these and other observations, hypoactivity in the nucleus accumbens, and interconnected circuitry, is hypothesized to contribute to the development and expression of cocaine addiction (Zhang et al, 1998; Jentsch and Taylor, 1999; Volkow and Fowler, 2000).

Although there are multiple lines of evidence for a role of hypoactivity in drug addiction (see above references for a review), the means by which it might actually contribute to the disorder are not understood. Cocaine addiction is defined by an increase in drug-directed behavior and a concomitant weakening of other motivated behaviors (American Psychiatric Association, 1994). Although the accumbens and interconnected structures facilitate drugdirected behavior, they also facilitate multiple alternative 
motivated behaviors (eg Everitt, 1990; Blackburn et al, 1992; Ikemoto and Panksepp, 1999; Cardinal et al, 2002; Kelley, 2004; Schwienbacher et al, 2004; Wise, 2004; Salamone et al, 2005). Given the selective changes in behavior that define addiction, it is difficult to envision how a general hypoactivity of the accumbens and interconnected circuits could contribute to drug addiction.

One potential explanation is that synapses and neurons that mediate drug-directed behavior undergo less cocaineinduced hypoactivity than do synapses and neurons and that contribute to other motivated behaviors, and moreover, that this differential hypoactivity mediates differential changes in drug- and non-drug-directed behaviors. This hypothesis is based on a number of previous observations. Numerous studies have shown that during cocaine selfadministration sessions, only a subset of accumbal neurons show changes in activity related to encoding aspects of the instrumental task (eg Carelli et al, 1993; Peoples et al, 1997, 1998b). The majority of those neurons show either transient or tonic increases in firing during the period of drug exposure (referred to as Task-Activated neurons). Other neurons, including those that mediate non-drug-related motivated behaviors (Bowman et al, 1996; Carelli, 2002; Deadwyler et al, 2004) do not show similar activations in activity during the drug session (referred to as Task-NonActivated neurons). Based on these observations, and evidence that accumbal neurons are subject to activitydependent neuroplasticity (Kombian and Malenka, 1994; Pennartz et al, 1993, 1994), we have hypothesized that the difference in activity between the Task-Activated and TaskNon-Activated neurons during periods of cocaine selfadministration is associated with a differential stimulation of signal transduction and gene transcription pathways between the two groups of neurons. This different pattern of molecular activity is hypothesized to make the TaskActivated neurons less susceptible than Task-Non-Activated neurons, to the adaptations that mediate hypoactivity. It may additionally make the Task-Activated neurons more susceptible to adaptations that either maintain or enhance activity. A relative sparing of neurons involved in drug seeking and taking from the inhibitory effects of repeated drug exposure would be associated with a relative increase in the transmission of signals related to drug-directed behavior, through the cortico-striato-thalamo-cortical circuit, and an absolute decrease in the transmission of signals related to other behaviors. This differential change in signaling would be expected to contribute to a selective increase in drugdirected behavior and a weakening of other motivated behaviors (referred to as the differential inhibition hypothesis, Peoples and Cavanaugh, 2003; Peoples et al, 2004).

A basic prediction of the hypothesis is that exposing animals to a history of cocaine self-administration will lead to less hypoactivity in Task-Activated neurons as compared to Task-Non-Activated neurons. This differential hypoactivity might be expressed in behaving animals as a smaller between-session decrease in basal firing rates of Task-Activated neurons relative to Task-Non-Activated neurons. The present study was designed to conduct an initial test of this prediction.

Rats were exposed to 30 daily long-access $(6 \mathrm{~h})$ cocaine self-administration sessions. Chronic extracellular recordings of the activity of individual accumbal neurons were made on days 2-3 and day 30 of the regimen. Betweensession comparisons of Task-Activated and Task-NonActivated neurons showed that the latter, but not the former, exhibited a decrease in basal firing rates. Additionally, during the day-30 session, the magnitude of the difference in firing rate between Task-Activated and Task-Non-Activated neurons was greatest in animals that showed the highest propensity to seek and take cocaine. The findings of the present study are consistent with a basic prediction of the differential inhibition hypothesis. Further investigation of the hypothesis, and more generally further investigation of differential drug-induced plasticity, might contribute to the discovery of novel mechanisms that contribute to drug addiction.

\section{METHODS}

\section{Subjects, Surgery, and Postoperative Maintenance}

Subjects were 38 male Long-Evans rats. Animals were anesthetized with sodium pentobarbital (50 mg/kg, i.p.). A catheter was implanted in the jugular vein and exited through a j-shaped stainless-steel cannula cemented to the skull. An array of 12-16 quad-Teflon coated stainless-steel wires was implanted in the accumbens (between 0.7 and $2.7 \mathrm{~mm}$ anterior from bregma; between 0.8 and $2.2 \mathrm{~mm}$ lateral from bregma; and between 6.8 and $7.2 \mathrm{~mm}$ ventral from level skull) (Paxinos and Watson, 1996). At least 7 days after surgery and 3 days before self-administration training, subjects were transferred to a Plexiglas $(37 \times 23 \times$ $\left.29 \mathrm{~cm}^{3} ; l \times w \times h\right)$ chamber where they remained $24 \mathrm{~h}$ a day for the duration of the study. Subjects had free access to water and were fed approximately $15 \mathrm{~g}$ of food each day in order to maintain body weight at $350 \mathrm{~g}$. All animal care and protocols were in accordance with the Guide for the Care and Use of Laboratory Animals published by the USPHS and approved by the Animal Care and Use Committee of Rutgers, The State University of New Jersey. Detailed descriptions of the surgical and maintenance procedures, and of the chambers used for housing and operant training, are provided in another report (Peoples, 2003).

\section{Behavioral Procedures}

Long-access cocaine self-administration session: sequence of events. The onset of each self-administration session was signaled by the illumination of a stimulus light and the insertion of the response lever into the chamber. The lever insertion was followed by an intravenous infusion of saline $(0.2 \mathrm{ml})$, a 7.5 -s tone, and the offset of the stimulus light for $40 \mathrm{~s}$. This collective sequence of events was referred to as the discriminative stimulus $\left(S^{D}\right)$.

Following the $S^{D}$, animals self-administered cocaine on a Fixed-Ratio 1 (FR 1) schedule. Each press of the lever was immediately followed by an i.v. infusion of cocaine solution $(0.7 \mathrm{mg} / \mathrm{kg} / 0.2 \mathrm{ml}$ infusion). The infusion was paired with a 7.5-s tone and termination of the stimulus light for 40 s. During the light-off period, presses on the lever had no programmed consequence. Each long-access session was limited in duration to either $6 \mathrm{~h}$ or 80 infusions. At the end of the self-administration session, the stimulus light was extinguished and the response lever was removed from the 
chamber. Self-administration training sessions were conducted 7 days per week.

Early and late self-administration sessions. Animals were exposed to 1-3 preliminary training sessions in which the lever-press response was acquired. Thereafter, animals were exposed to a 30-day regimen of daily long-access cocaine self-administration sessions. Electrophysiological recordings were conducted on the second to third day (ie Early session) and the 30th day (ie Late session) of the regimen. Whether animals were included in either or both the Early and the Late sessions depended on catheter patency, function of electrophysiological equipment, and equipment availability on days of interest.

\section{Electrophysiological Recording Sessions}

Early session. The Early recording session started with a 20-min non-drug, baseline, recording period. During the non-drug baseline period, subjects were not exposed to the drug-associated cues, the response lever, or the drug. At the end of the 20-min period, the typical daily selfadministration session was conducted. Following completion of the self-administration phase, recording was continued for an additional 60-min recovery period. During this period, drug was no longer available and all cues related to self-administration, including the response lever, were absent.

Late session. The Late recording session was conducted in the same manner as the Early session except that the self-administration phase was followed immediately by an extinction and cue reinstatement procedure (deWit and Stewart, 1981). During the extinction phase of this procedure, the response lever remained in the chamber but lever presses had no consequence. The extinction phase continued until animals ceased responding for a minimum of $20 \mathrm{~min}$. Thereafter, the cue reinstatement phase was initiated. At the onset of this phase, animals were exposed to a reinstating cue $\left(\mathrm{C}^{\mathrm{R}}\right)$, which consisted of the light and tone cues that were typically associated with drug infusions. Lever presses had no consequence during the cue reinstatement phase.

Electrophysiological recording equipment and procedures. Activity from each recorded microwire was first led into a field effect transistor in the headstage of the electronic harness (NB Labs, Denison, TX). The neural signal was then led through a modified fluid and electronic swivel (CAY675-24, Airflyte Electronics, Bayonne, NJ) to a preamplifier (Riverpoint Electronics, Goldsboro, NC) that differentially amplified the signal on the recording wire against another microwire. The signal was then led through a bandpass filter $(450 \mathrm{~Hz}-10 \mathrm{kHz})$ and amplifier (Riverpoint Electronics, Goldsboro, NC). Using software and hardware of DataWave Technologies Inc. (Longmont, CO), electrical signals were sampled $(50 \mathrm{kHz}$ sampling frequency for each recording wire), digitized, time-stamped (0.1 ms resolution), and stored for offline analysis. After the experiment, cluster analysis software (Plexon Inc., Dallas, TX) was used to discriminate neural waveforms. After a population of waveforms was isolated by the discrimination procedures, it was subjected to an inter-spike interval histogram analysis to confirm that it corresponded to a discriminated single neuron (Peoples and West, 1996; Peoples, 2003). All neurons showed evidence of a refractory period, consistent with discrimination of waveforms associated with a single neuron (typically $\geqslant 3.0 \mathrm{~ms}$ ). Minimum signal: noise ratio of sorted units included in the study was $3: 1$.

\section{Analysis of Cocaine Intake during the Individual Self-Administration Sessions}

Rate of cocaine self-administration was characterized by determining the median inter-infusion-interval during maintenance (presses 20-30). Additionally, cocaine levels during this period were calculated using the pharmacokinetic equation for intravenous cocaine described by Pan et al (1991). Cocaine level at the time of each lever press was calculated with a $0.1 \mathrm{~min}$ temporal resolution by inputting body weight of the animal, cocaine dose per infusion, and intervals lapsing between successive lever presses into the equation. Calculations were made using software provided by Drs J Justice (Emory University) and Mark S Kleven (University of Pennsylvania). These measures were used as indices of the self-administration behavior of the individual animals and subjected to analyses of variance (ANOVA) designed to make between-session comparisons of cocaine-taking behavior.

\section{Analysis of Electrophysiological Data}

Firing patterns exhibited by individual neurons.

Overview: To test the prediction of interest, it was necessary to identify Task-Activated and Task-Non-Activated neurons during the Early and Late sessions. Previous studies documented that accumbal neurons exhibit multiple types of firing patterns during the self-administration session (Carelli et al, 1993; Chang et al, 1994; Peoples and West, 1996; Peoples et al, 1998b, 2004; Uzwiak et al, 1997). These firing patterns include the following: (1) changes in firing time-locked to events that occur at the onset of the self-administration session; (2) changes in firing time-locked to the cocaine-reinforced lever press; and (3) changes in average, tonic, firing that occur during the selfadministration session relative to the pre- and postsession baseline periods. These firing patterns were identified in the present study according to previously established statistical criteria (Peoples and West, 1996; Uzwiak et al, 1997; Peoples et al, 1998b, 2004), which are described below. As a frame of reference for comparisons to investigations that use percent change criteria (eg Hollander and Carelli, 2005; Chang et al, 1994), the statistical criteria used to test for task-related firing responses were moderately more stringent than a percent-change rule of $100 \%$.

Statistical criteria used to identify specific firing patterns: Changes in firing time-locked to events at the onset of the session. An $S^{\mathrm{D}}$ firing pattern was defined as a significant change in firing rate between the $30-s$ pre- $S^{\mathrm{D}}$ and the $30-s$ post- $S^{\mathrm{D}}$ (eg Figure 1a). To compare firing pre- and post- $S^{\mathrm{D}}$, number of discharges was determined as a function of 1-s bins. The number of discharges in the 30, 1-s bins, 

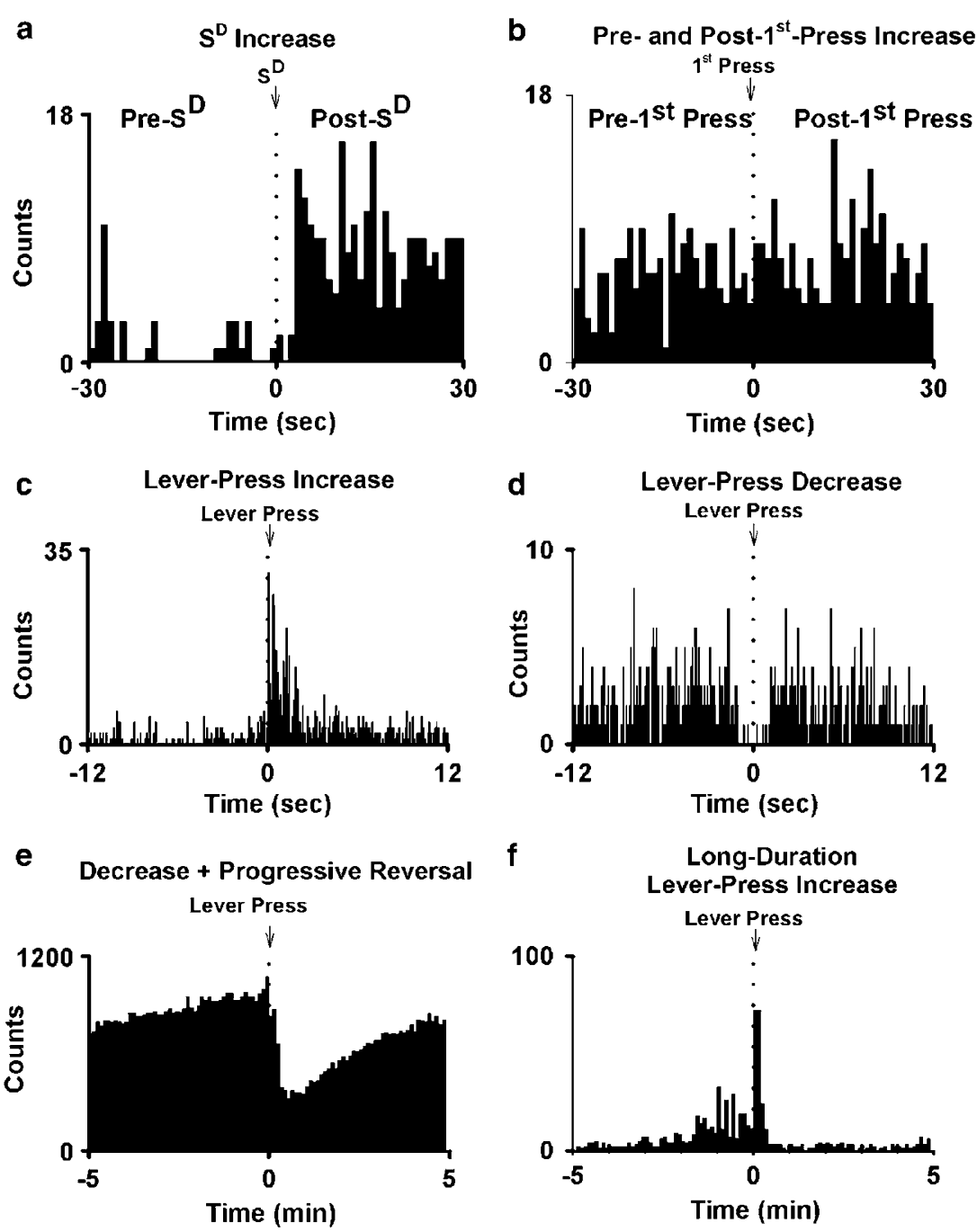

g

Session Increase

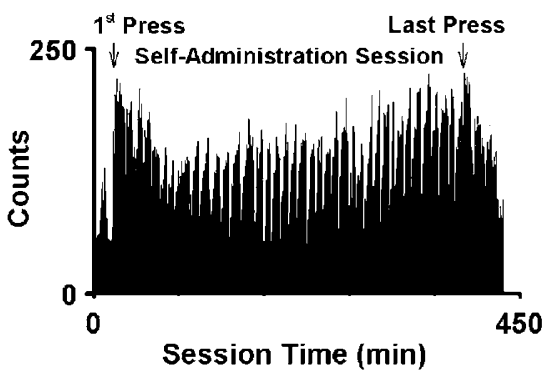

h

Session Decrease

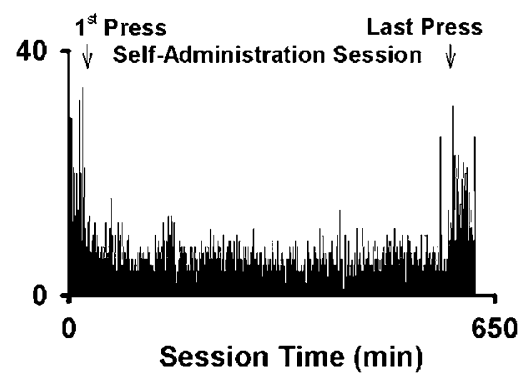

Figure I Single neuron examples of firing patterns exhibited by accumbal neurons during the Early and the Late sessions. All the histograms shown in Figure I display the activity of neurons recorded during the Early session; however, the firing patterns are representative of those observed during both the Early and the Late session. ( $a$ and $b$ ) Panels $a$ and $b$ show firing patterns of an individual neuron that exhibited an excitatory $S^{D}$ firing pattern $(a)$, an excitatory pre-first-press firing pattern (compare $b$ to $a$ ), and an excitatory post-first-press firing pattern (compare $b$ to $a$ ). In (a), number of discharges (counts) per $\mathrm{I}$-s bin is plotted as a function of time before and after the $S^{D}$ and in (b), the same measure of firing is plotted pre- and post-first-press. (c and d) Panels show an excitatory short-duration lever-press firing pattern and an inhibitory short-duration lever-press firing pattern. In each histogram, counts per 0.1 -s bin are plotted for the $12 \mathrm{~s}$ pre- and postpress. (e and f) Panels show a decrease + progressive-reversal firing pattern and a long-duration lever-press firing pattern. In each histogram, firing rate (ie counts per 0.1 -min bin) is plotted for the 5 min pre- and post-press. (Panels $g$ and h) Panels show a session-increase firing pattern and a session-decrease firing pattern. For each histogram, firing rate (ie counts per 0.5-min bin) is plotted as a function of time in the session.

pre- $S^{\mathrm{D}}$ were then compared to the number of discharges in the 301 -s bins post- $S^{\mathrm{D}}$, using a Mann-Whitney test $(\alpha=0.05$, two-tailed test). Pre- and post-first-press responses were similarly defined and analyzed (eg Figure 1a and b). Specifically, a significant pre-first-press response was defined as a significant change in firing during the $30 \mathrm{~s}$ immediately preceding the first press, relative to the $30 \mathrm{~s}$ pre- $\mathrm{S}^{\mathrm{D}}$. A post-first-press response was defined as a 
significant change in firing during the $30 \mathrm{~s}$ after the first press relative to the $30 \mathrm{~s}$ pre- $S^{\mathrm{D}}$.

Changes in firing time-locked to the reinforced lever press. Both short and long-duration changes in firing were observed to occur time-locked to the cocaine-reinforced lever press. Both types of firing patterns were identified using lever presses that occurred during the maintenance phase of the self-administration session, when cocaine levels and response rates were stable. The maintenance phase included all reinforced presses except for the first 10 .

A short-duration lever-press firing pattern was defined as either a significant increase or decrease in average firing rate within either the $3 \mathrm{~s}$ pre-press or the $3 \mathrm{~s}$ post-press, relative to firing during the -12 to $-9 \mathrm{~s}$ pre-press (eg Figure $1 \mathrm{c}$ and $\mathrm{d}$ ). To test for a difference in firing between these two intervals, firing rate (ie number of discharges) during the $12 \mathrm{~s}$ pre- and post-press was determined using a sliding window method with a window of $0.3 \mathrm{~s}$ and a step of $0.1 \mathrm{~s}$. The window with the apical number of discharges during the $\pm 0.3 \mathrm{~s}$ of the press and the window with the median number of discharges during the -12 to $-9 \mathrm{~s}$ pre-press were identified. The number of discharges that occurred during those two windows was then determined on trial-by-trial basis. The numbers for each trial were input as pairs into a Wilcoxon matched pairs test (one-tailed, $\alpha=0.01$ ).

Long-duration lever-press firing patterns (Figure 1f) were similar to short-duration lever-press patterns except that they were longer in duration. To test for a long-duration lever-press firing pattern, the statistical comparison was made between a 30 -s window pre-press and a 30-s window post-press. These windows were determined using a sliding window method with a window of $30 \mathrm{~s}$ and a step of $6 \mathrm{~s}$. For most neurons, the change in firing began and ended within either the $1.0 \mathrm{~min}$ pre-press or the $1.0 \mathrm{~min}$ post-press. For other neurons, the change began within the $1.0 \mathrm{~min}$ post-press, and recovered slowly, over the course of the inter-infusion interval (referred to as decrease + progressive-reversal firing pattern; Figure 1e).

Session-change in firing. A session change in firing consisted of a change in average, tonic, firing during the entire self-administration session, relative to baseline periods that preceded and followed the session. The firing patterns were statistically defined by a significant change in the last $20 \mathrm{~min}$ of the self-administration session relative to the last $20 \mathrm{~min}$ of the pre-session baseline phase of the recording session. To test for the firing pattern, number of discharges was calculated as a function of $0.5 \mathrm{~min}$ bins from the onset to the offset of the recording session. The discharges during the 40 bins of the baseline period were compared to the discharges during the last 40 bins of the self-administration session, using a Mann-Whitney test (one-tailed test, $\alpha=0.05$; Figure $1 \mathrm{~g}$ and $\mathrm{h}$ ).

Between-session comparisons of Task-Activated and TaskNon-Activated neurons. It was predicted that neurons that were activated, either transiently or tonically, during periods of cocaine exposure, would show less betweensession inhibition than would neurons that showed no such activation (see Introduction). To test the hypothesis, neurons were sorted into two groups: (1) those that showed at least one excitatory change in firing during the selfadministration session (referred to as Task-Activated) and
(2) those that showed no excitatory change in firing during the self-administration session (referred to as Task-NonActivated). This sort formed two groups that differed on the independent variable of interest (activation vs no activation).

To characterize the selectivity of between-session changes in firing, multiple time points during each recording session were included in the comparison of Task-Activated and Task-Non-Activated neurons. The time points included the following: (1) the $30 \mathrm{~s}$ pre- $\mathrm{S}^{\mathrm{D}}$ (ie measure of basal firing), (2) the $30 \mathrm{~s}$ post- $S^{\mathrm{D}}$, (3) the $30 \mathrm{~s}$ pre-1st-press, (4) the $30 \mathrm{~s}$ post-1st-press, and (5) the 30-s pre-press period during which the median pre-press firing rate for presses 20-30 was achieved (see Peoples et al, 1998a, 2004; Peoples and Cavanaugh, 2003). The firing rates during these periods were subjected to an ANOVA (two-tailed, $\alpha=0.05$ ). A number of additional control analyses were conducted to confirm that any differential between-session change in the firing of Task-Activated and Task-Non-Activated neurons reflected a general difference between activated and nonactivated neurons rather than a difference between particular subsets of those larger groups of neurons (see Control analyses in Results).

Preliminary graphical examination of firing patterns suggested that the spike counts should be transformed prior to formal analyses, as the distributions of counts were skewed toward higher values. We used the transformation of $\log _{10}(x+1)$, which reduced the skewness to appropriate levels.

Only five of the microwires that were used in the Early and the Late session were used in both sessions. It is possible that some of the recordings made with the five wires during the Early and Late sessions were of the same neurons. However, previous studies have demonstrated that it is unlikely that this was true for all, even most, of the wires (cf, Peoples et al, 1999; Peoples, 2003). The neurons recorded during the Early and the Late session were thus treated as independent in all statistical analyses.

Relationship between cocaine-seeking behaviors and firing of Task-Activated and Task-Non-Activated neurons. It is hypothesized that differential changes in the firing of Task-Activated and Task-Non-Activated neurons contributes to increments in drug seeking and taking that occur with repeated exposure to cocaine self-administration session. If this hypothesis were correct, one might expect that there would be a positive relationship between the difference in firing between the two groups of neurons and the propensity of animals to seek and take drug. To test this prediction, the firing of Task-Activated and Task-NonActivated neurons was compared during the Late session in groups of animals that differed in operant response rates during extinction and cue reinstatement.

Animals were ranked by the number of presses that they made during the extinction and cue reinstatement phases of the Late session. The animals that were in the top $1 / 3$ of both phases were defined as the high seekers. All other animals were defined as low-moderate seekers. The strategy for differentiating between high and low-moderate seekers is consistent with that employed by other researchers (Deroche-Gamonet et al, 2004) and allowed inclusion of all 
neurons and animals in the statistical analysis. The difference in the firing rates of Activated and Non-Activated neurons was compared between the high and low-moderate cocaine seekers. Average firing rates of Task-Activated and Task-Non-Activated neurons were subjected to a threefactor ANOVA with cocaine seekers, activation and session phase as factors. The session phases were those included in the original analysis, as well as a $30-\mathrm{s}$ period (min $29.5-30)$ during the extinction phase, and the $30 \mathrm{~s}$ before and after the start of cue reinstatement (ie $30 \mathrm{~s}$ pre- and post- $\mathrm{C}^{\mathrm{R}}$ ). For all animals, the $29.5-30 \mathrm{~min}$ period of the extinction phase corresponded to an active period of lever pressing. The $30 \mathrm{~s}$ pre- $\mathrm{C}^{\mathrm{R}}$ period occurred $20 \mathrm{~min}$ after the last extinction response. The additional time points were incorporated into the analysis given that the two groups were formed on the basis of behavior during those time periods, and to test for a potential effect of those procedures on the difference in firing between activated and nonactivated neurons.

Between-session comparisons of particular firing patterns. In addition to testing for overall between-session changes in average firing, it was of interest to test for between-session changes in the phasic firing patterns of Task-Activated neurons. Evidence from studies of instrumental behavior reinforced by non-drug rewards shows that the prevalence and magnitude of phasic firing patterns related to task events can change across repeated sessions, in association with learning (see Discussion). Such changes could potentially occur in animals exposed to repeated daily self-administration sessions. Additionally, phasically activated neurons show increased rates of firing during specific task-related periods relative to other periods in the session. In parallel with predictions related to overall firing rates of Task-Activated and Task-Non-Activated neurons, one might expect that firing of the Task-Activated neurons during task and non-task periods might undergo differential between-session changes, such that the activity during the former periods would be enhanced relative to the latter (see Discussion). Based on these possibilities, we tested for an effect of session on each of the more common firing patterns. These firing patterns included all firing patterns except for the long-duration lever-press firing patterns and task-inhibited firing patterns (eg $S^{D}$ decrease or lever-press decrease), which were too few in number to subject to statistical analyses. Due to the partial overlap among neurons that exhibited the various firing patterns, it was necessary to conduct a separate ANOVA for each of the firing patterns. The ANOVA included two factors: (1) session (ie Early vs Late) and (2) time intervals that defined a particular firing pattern (eg the $-30 \mathrm{~s}$ pre $-S^{D} v s$ the $30 \mathrm{~s}$ post- $S^{\mathrm{D}}$ ).

\section{Histology}

Subjects were injected with a lethal dose of sodium pentobarbital. Anodal current $(50 \mu \mathrm{A}$ for $4 \mathrm{~s})$ was passed through each microwire. Animals were perfused with formalin-saline. Coronal sections $(50 \mu \mathrm{m})$ were mounted on slides and incubated in a solution of $5 \%$ potassium ferricianide and $10 \% \mathrm{HCl}$ to stain the iron deposits left by the recording tip (Green, 1958). The tissue was counter- stained with $0.2 \%$ solution of Neutral Red. The location of each wire tip was plotted on the coronal plate (Paxinos and Watson, 1996) that most closely corresponded to its anterior-posterior position. Each neuron included in the study was verified to be within the accumbens and was further localized either to the core, the shell, or the core-shell border.

\section{RESULTS}

\section{Patterns of Drug Intake}

The pattern of drug intake exhibited by all subjects that were included in the Early (ie 28) and the Late session (ie 19) are shown in Figure 2. Statistical analyses (see Supplementary Materials) showed that animals increased the rate of cocaine intake and maintained a higher cocaine level during the Late session relative to the Early session. This escalation in cocaine taking is consistent with that observed in other studies employing a comparable cocaine self-administration regimen (eg Ahmed and Koob, 1998).

\section{Neural Data}

A total of 82 and 49 microwires were used to record activity of 87 and 55 accumbal neurons during the Early and the Late session, respectively. The majority of the microwires were located in either the core or the medial portions of the shell (see Supplementary Figure S1).
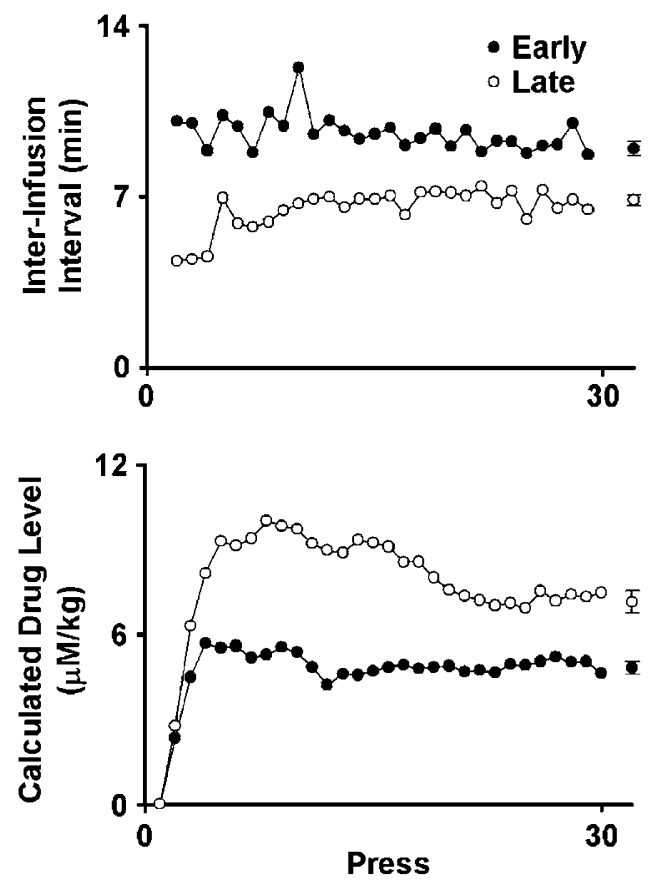

Figure 2 Average rate of drug intake for all the subjects during the Early and the Late sessions. Top: Average interinfusion-interval (ie min between successive self-infusions) is plotted as a function of press number. Bottom: Average calculated drug level $(\mu \mathrm{M} / \mathrm{kg})$ at the time of the press is plotted as a function of lever-press. To the right of each plot is shown the average median value $( \pm S E)$ for the last 10 cocaine-reinforced lever presses. Error bars are shown for only the averages subjected to statistical analyses. 
Table I Percent of All Recorded Neurons that Showed Excitatory Responses to Particular Task Events is Shown for the Early and the Late Session

Firing pattern

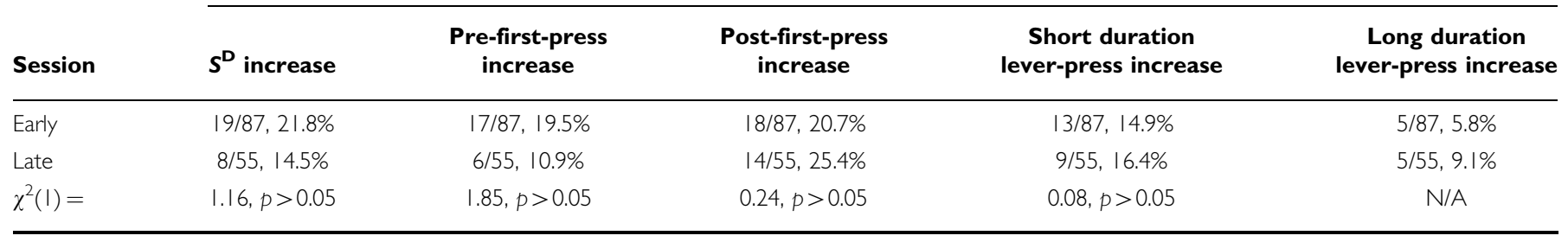

$\mathrm{N} / \mathrm{A}$ indicates that $\mathrm{N}$ was too small for statistical comparison. Some neurons exhibited more than one firing pattern and therefore contributed to more than one prevalence estimate.

Table 2 Percent of All Recorded Neurons that Exhibited Decrease+Progressive-Reversal, Session-Decrease, and SessionIncrease Firing Patterns is Shown for the Early and the Late Session

\begin{tabular}{lccc}
\hline & \multicolumn{3}{c}{ Firing pattern } \\
\cline { 2 - 4 } Session & $\begin{array}{c}\text { Decrease+Progressive } \\
\text { reversal }\end{array}$ & $\begin{array}{c}\text { Session } \\
\text { decrease }\end{array}$ & $\begin{array}{c}\text { Session } \\
\text { increase }\end{array}$ \\
\hline Early & $36 / 87,41.4 \%$ & $51 / 87,58.6 \%$ & $19 / 87,21.8 \%$ \\
Late & $\mid 4 / 55,25.5 \%$ & $33 / 55,60.0 \%$ & $15 / 55,27.3 \%$ \\
$\chi^{2}(I)=$ & $3.7, p=0.05$ & $0.03, p>0.05$ & $0.54, p>0.05$
\end{tabular}

\section{Between-Session Comparisons of Particular Firing Patterns}

There were no significant changes in the prevalence of any of the particular firing patterns (Figure 1) that were compared between the Early and the Late sessions, though there was a trend for the decrease + progressive reversal pattern to be less common during the Late session than during the Early session (Tables 1 and 2). The neurons that showed particular types of inhibitory task-related firing patterns during the Early and the Late session were too few in number to be compared separately. However, there was no between-session change in the prevalence of all inhibitory firing patterns combined $\left(\chi^{2}<1.0\right)$. For each subgroup of neurons that exhibited a particular type of firing pattern, between-session comparisons were made of the average firing rates during the intervals that defined the firing pattern (see Methods). The ANOVAs showed that there were no significant between-session changes in the average firing patterns (Figures 3 and 4), although there was a nonsignificant trend for the session-increase neurons to show a between-session increase in overall firing rate (Figure 4c, Table 3).

\section{Between-Session Comparisons of Task-Activated $v s$ Task-Non-Activated Neurons}

Prevalence. Consistent with the between-session stability in the prevalence of excitatory firing patterns, the total number of neurons that were classified as Task-Activated and Task-Non-Activated did not change between the Early and the Late session $\left(\chi^{2}<1.0\right)$. Specifically, during the Early

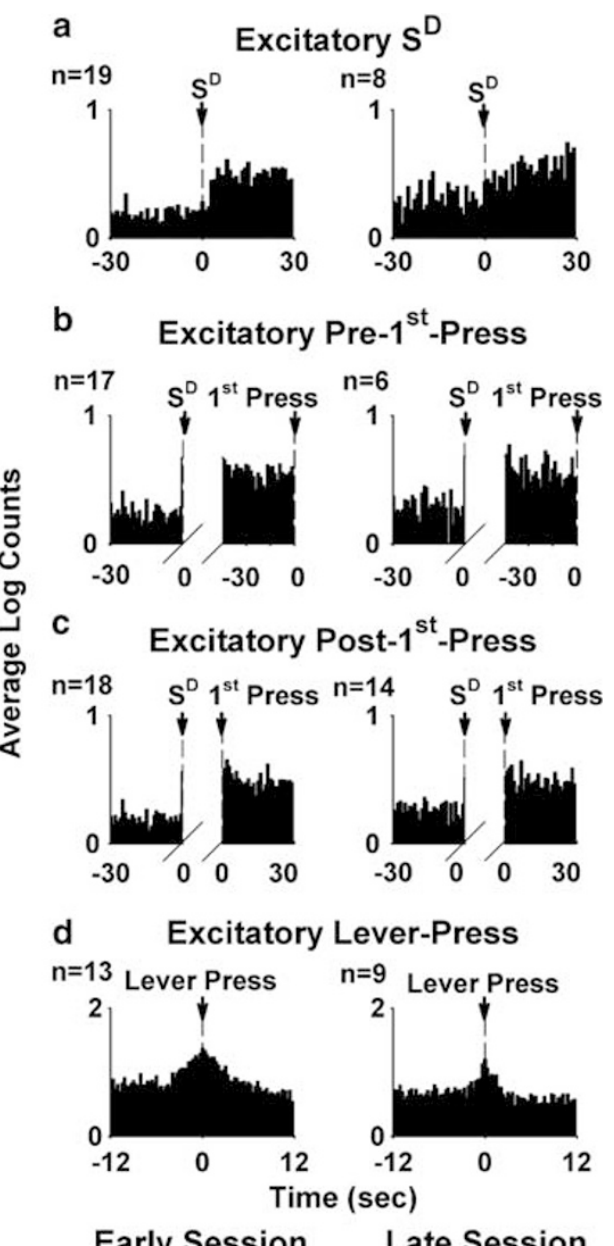

Early Session Late Session

Figure 3 Population histograms of all neurons that showed particular excitatory task-related firing patterns. The left and right columns of the figure show firing patterns observed during the Early and the Late sessions, respectively. (a) Average firing during the 30 s pre- and post- $S^{D}$ is plotted for all neurons that showed an excitatory $S^{D}$ firing pattern. (b) Average firing during the $30 \mathrm{~s}$ pre- $\mathrm{S}^{\mathrm{D}}$ and the $30 \mathrm{~s}$ pre-first press is plotted for all neurons that showed an excitatory pre-first-press firing pattern. (c) Average firing during the $30 \mathrm{~s}$ pre- $\mathrm{S}^{\mathrm{D}}$ and the $30 \mathrm{~s}$ post-first press is plotted for all neurons that showed an excitatory post-first-press firing pattern. In each of the histograms shown in $(a-c)$, bin width is $1.0 \mathrm{~s}$. (d) Average firing during the $12 \mathrm{~s}$ pre- and post-press is plotted for all neurons that showed an excitatory short-duration lever-press firing pattern ( $0.3 \mathrm{~s}$ bin width). The N's noted in the figure correspond to the total number of neurons that exhibited particular firing patterns. Because neurons often showed more than one excitatory task-related firing pattern, some individual neurons are represented in more than one population histogram. 

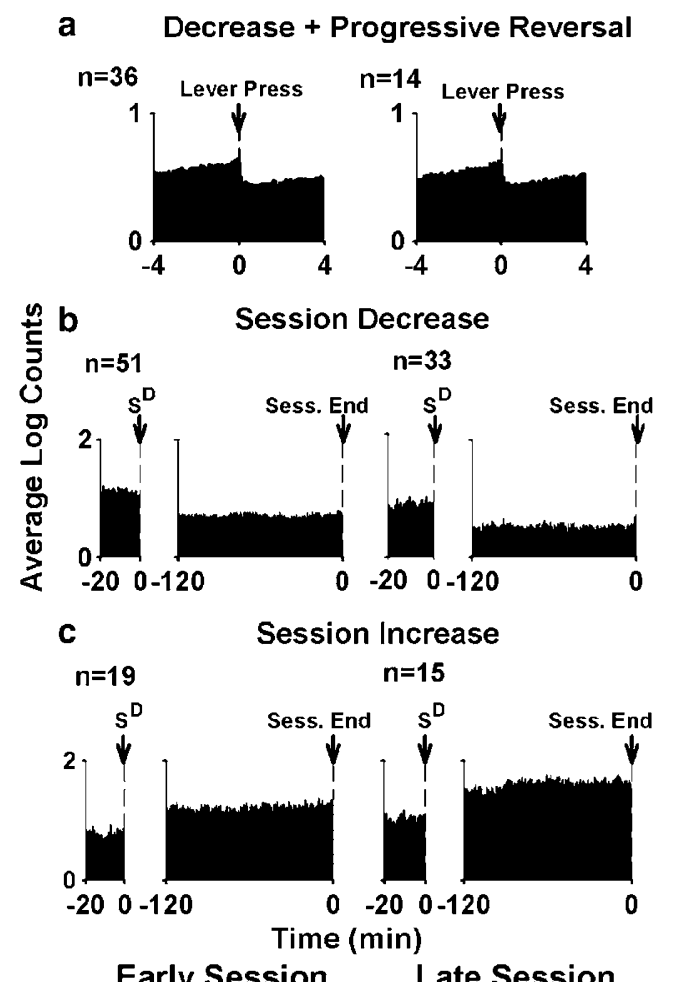

Figure 4 Population histograms of all neurons that showed either a decrease + progressive-reversal firing pattern, a session-decrease firing pattern, or a session-increase firing pattern. (a) Average firing rate during the $4 \mathrm{~min}$ pre- and post-press is plotted for all neurons that showed a decrease + progressive-reversal firing pattern during the Early (left) and the Late (right) sessions. The measure of average firing equals average per 0.1 min bin. ( $b$ and c) Average firing rate plotted as a function of time during the recording session for all neurons that showed either a session-decrease firing pattern (b) or a session-increase firing pattern (c). In (b) and (c), average firing equals average per 0.5 - min bin and is plotted during the last 20 min pre- $S^{D}$ and the last $2 h$ of the self-administration session. Because neurons often showed more than one excitatory task-related firing pattern, some individual neurons are represented in more than one population histogram.

Table 3 ANOVA Results for between-Session Comparisons of Particular Firing Patterns are Shown

ANOVA factors and F-test

\begin{tabular}{lcc}
\cline { 2 - 3 } Firing pattern & Session & Session $\times$ Event time \\
\hline$S^{D}$ increase & $F(I, 25)<1.0$ & $F(I, 25)<1.0$ \\
Pre-first-press increase & $F(I, 2 I)<1.0$ & $F(I, 21)<1.0$ \\
Post-first-press increase & $F(I, 30)<1.0$ & $F(I, 30)=1.88, p>0.05$ \\
Short-duration lever & $F(I, 20)<1.0$ & $F(I, 20)=3.16, p>0.05$ \\
press increase & & \\
Decrease+Progressive & $F(I, 48)=1.09, p>0.05$ & $F(I, 48)<1.0$ \\
reversal & & \\
Session decrease & $F(I, 82)=1.88, p>0.05$ & $F(I, 82)<1.0$ \\
Session increase & $F(I, 32)=3.43, p=0.07$ & $F(I, 32)<1.0$ \\
\hline
\end{tabular}

and the Late session, 52\% (45/87) and 57\% (31/55) of the neurons were identified as Task-Activated. During the same sessions, $48 \%(42 / 87)$ and $43 \%(24 / 55)$ of the neurons were categorized as Task-Non-Activated.

Between-session comparisons of the average firing rates of the Task-Activated vs Task-Non-Activated neurons.

Primary comparison: Comparison of the firing rates of the Task-Activated and Task-Non-Activated neurons showed that the two groups exhibited a significantly different between-session change in firing. Moreover, this differential change in firing was apparent in comparisons of all phases of the Early and Late recording sessions (Figures 5 and 6). An ANOVA showed that there was no significant three-way interaction between activation (ie Task-Activated vs Task-Non-Activated), session, and session phase $(\mathrm{F}(4,140)<1.0)$, and no significant two-way interaction between session and session phase $(\mathrm{F}(4,140)<1.0)$. There was a significant two-way interaction between activation and session phase $(\mathrm{F}(4,140)=8.49, p<0.0001)$, and between activation and session $\mathrm{F}(1,140)=7.39, p=0.007)$. Additional between-session contrasts showed that between the Early and the Late session, average firing of the TaskActivated neurons did not change significantly; whereas, firing of the Task-Non-Activated neurons showed a significant decrease $(p=0.02)$. Due to the heterogeneous between-session change in firing the difference $(p=0.007)$ and the ratio between the average firing of the TaskActivated and Task-Non-Activated neurons increased between the Early (difference in $\log _{10}$ counts $=0.33 \pm 0.10$ ) and the Late session (difference in $\log _{10}$ counts= $0.78 \pm 0.14)$.

Control analyses: There were no differential betweensession changes in sampling of Task-Activated and TaskNon-Activated neurons. Control analyses showed that the differential between-session change in firing rate was not attributable to a between-session change in the sampling of Task-Activated and Task-Non-Activated neurons from the core $v s$ the shell. Similarly, the differential between-session change in firing was not due to a differential sampling of neurons across different animals (see Supplementary Materials).

The absence of a between-session decrease in firing was a consistent characteristic of Task-Activated neurons. The trend for session-increase neurons to show a between-session increase in firing (Figure $4 \mathrm{c}$, Table 3, described above) suggested the possibility that the differential between-session inhibition of Task-Activated and Task-Non-Activated neurons was due primarily to the unique inclusion of sessionincrease neurons in the Task-Activated group. To test for this possibility, the original ANOVA comparing TaskActivated and Task-Non-Activated neurons was repeated with the activation factor redefined so that it had three levels: (1) session-increase neurons, (2) all other TaskActivated neurons (referred to as Task-But-Not-SessionActivated neurons), and (3) all Task-Non-Activated neurons.

The ANOVA showed that there was no significant threeway interaction between activation, session, and session phase $(\mathrm{F}(8,140)<1.0)$. There was also no two-way interaction between session and session phase $(F(4,140)<1.0)$. There was a significant interaction between activation and session $(\mathrm{F}(2,140)=4.84, p=0.009)$ (Figure 7). Betweensession contrasts showed that between the Early and the Late session, average firing underwent a significant decrease for the Task-Non-Activated neurons $(p=0.01)$ and no significant change for the Task-But-Not-Session-Activated neurons $(p=0.98)$. There was a trend for session-increase 


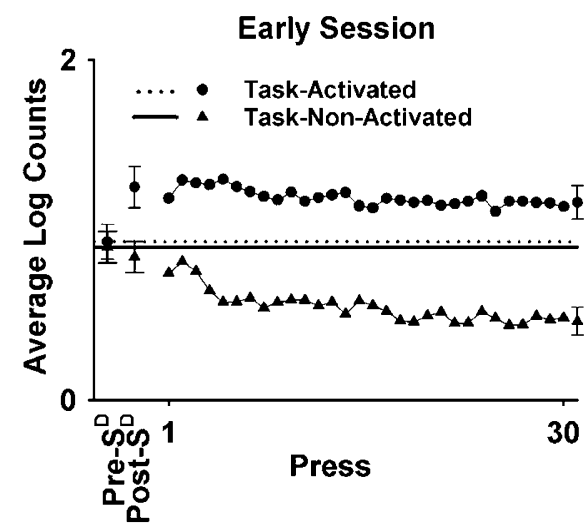

Late Session

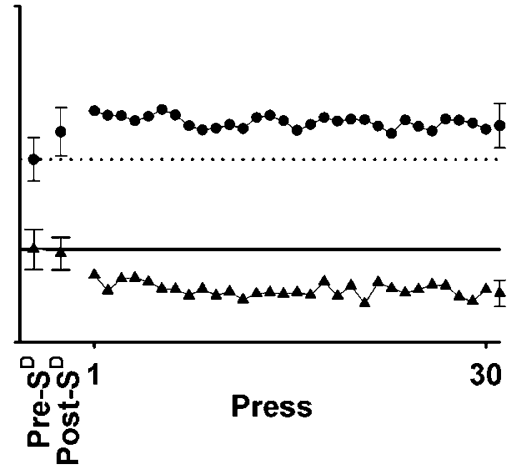

Figure 5 Average firing of Task-Activated and Task-Non-Activated neurons during the Early and the Late session. Average firing of the Task-Activated and Task-Non-Activated neurons during various periods of the self-administration session is plotted for the Early (left plot) and the Late (right plot) session. The periods for which firing is shown include: (I) 30 s pre- $S^{D}$, (2) 30 s post- $S^{D}$, and (3) 30 s pre-press for each of the first 30 cocaine-reinforced lever presses. For pre- $S^{D}$ and post- $S^{D}$, the standard error of the mean is also presented. To the immediate right of each graph is shown average median maintenance prepress firing $( \pm S E)$. Average pre- $S^{D}$ firing rates are shown as horizontal lines, as well as points on the graph, to facilitate between- and within-session comparisons.

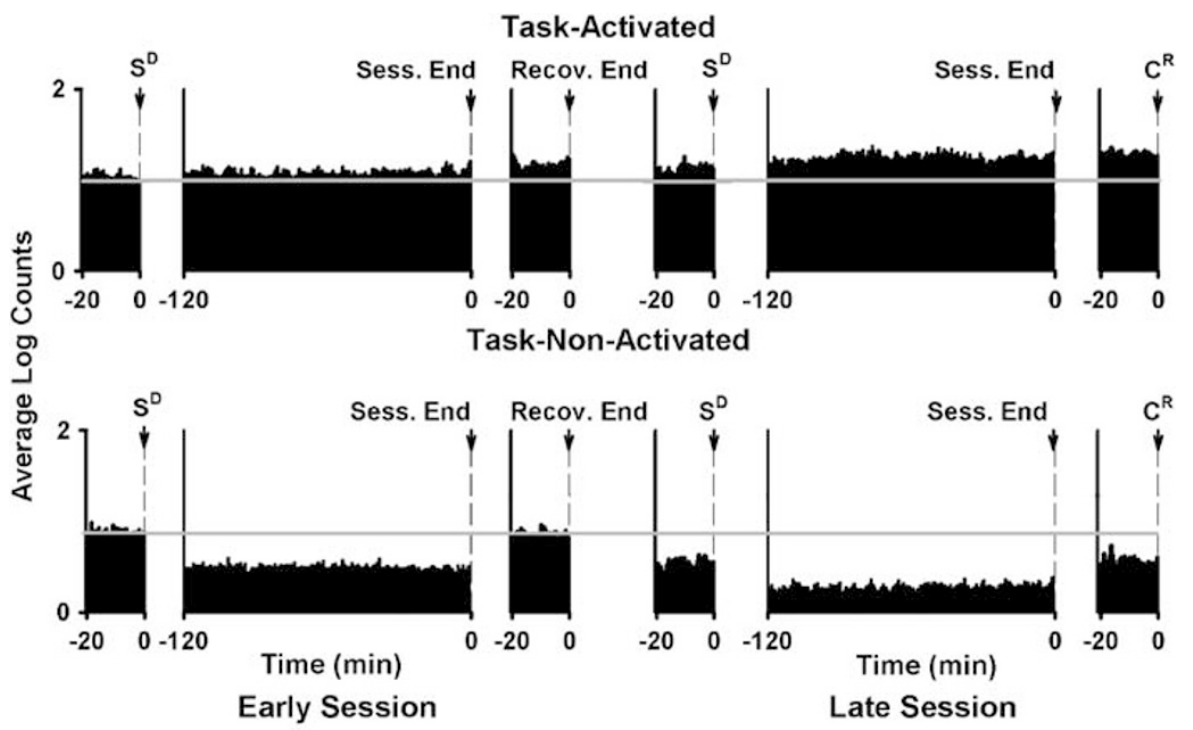

Figure 6 Population histograms showing the average baseline and self-administration firing rates exhibited by Task-Activated and Task-Non-Activated neurons during the Early and the Late sessions. Average firing of the Task-Activated neurons (top) and Task-Non-Activated neurons (bottom) during the Early session (left) and the Late session (right) are shown for the following periods: (I) 20 min pre- $S^{\mathrm{D}}$, (2) the last $2 \mathrm{~h}$ of the self-administration session, and (3) the last $20 \mathrm{~min}$ of either the Recovery period (Early session) or the $20 \mathrm{~min}$ pre- $C^{\mathrm{R}}$ (Late session). The solid gray horizontal lines across the histograms correspond to the average pre- $S^{D}$ firing rate during the Early session and is shown to facilitate between-session comparisons of average firing rates. Note that in the present figure average firing rate is plotted as a function of successive 0.5 min periods and is locked to phases of the session. This contrasts with Figure 6 in which average firing is locked to specific task events.

neurons to show an increase in firing between the Early and Late session, but this trend did not reach statistical significance $(p=0.05)$. These results demonstrate that the differential decrease in average firing of Task-Activated and Task-Non-Activated neurons was not due to the unique inclusion of session-increase neurons in that group of neurons and that it instead reflected a general characteristic of neurons that were activated during the self-administration session.

In line with the above interpretation, there was no evidence of a between-session decrease in average baseline firing rates for any of the transiently activated subgroups of Task-Activated neurons (Figure 8). As already described, the average firing patterns of the transiently activated neurons also remained stable between the Early and the Late session. Together these data are consistent with the interpretation that an absence of a between-session decrease in firing was a consistent characteristic across subtypes of Task-Activated neurons (for additional relevant control analyses see Supplementary Materials).

The differential between-session decrease in firing of Task-Activated and Task-Non-Activated neurons was a differential change in overall firing rather than a differential change in firing during specific task periods: The lack of a significant effect of session phase on the differential between-session change in the firing of 

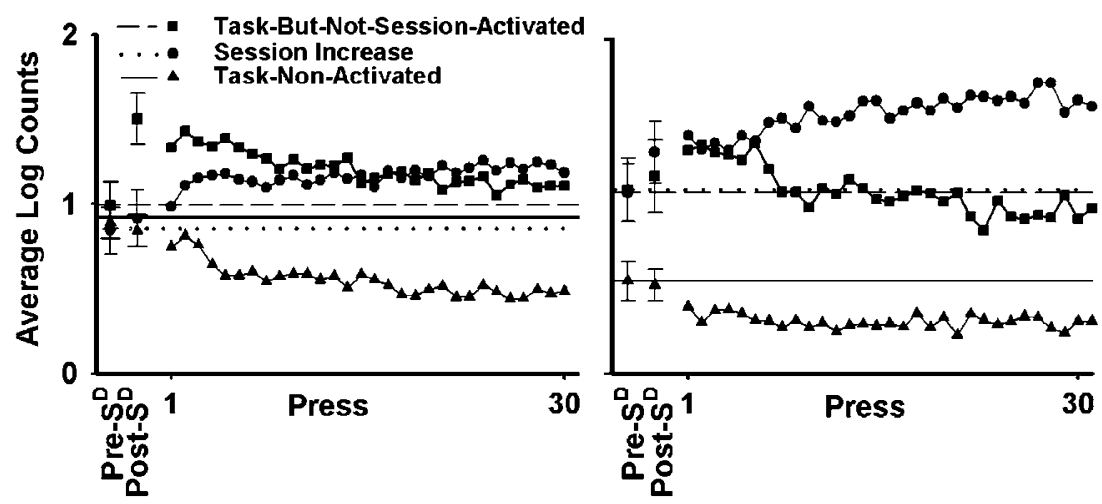

Figure 7 Average firing of Session-Increase, Task-But-Not-Session-Activated, and Task-Non-Activated neurons during the Early and the Late session. Average firing of the Session-Increase, Task-Activated and Task-Non-Activated neurons during various periods of the self-administration session is plotted for the Early (left plot) and the Late (right plot) session. The periods for which firing is shown include: (I) $30 \mathrm{~s} \mathrm{pre-S^{D }}$, (2) $30 \mathrm{~s}$ post- $S^{\mathrm{D}}$, and $(3) 30 \mathrm{~s}$ pre-press for the first 30 cocaine-reinforced lever presses. For pre- $S^{D}$ and post- $S^{\mathrm{D}}$, the standard error of the mean is also presented. Average pre- $S^{\mathrm{D}}$ firing rates are shown as horizontal lines, as well as points on the graph, to facilitate between- and within-session comparisons.

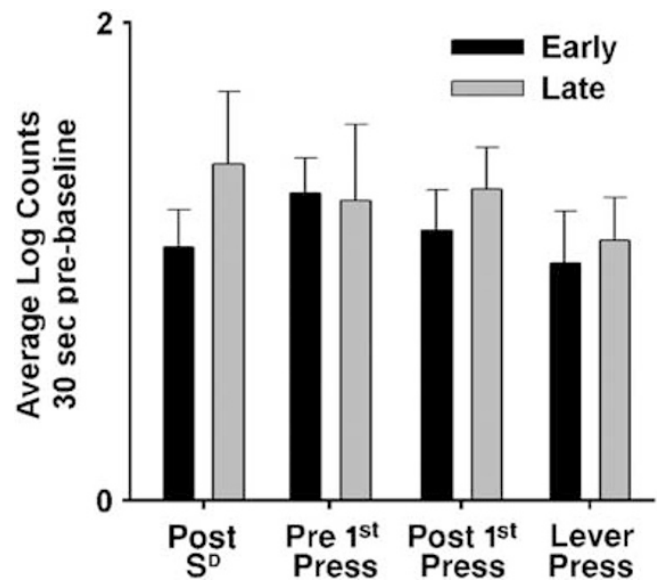

Figure 8 Average pre- $S^{D}$ firing rates of the transient subtypes of TaskActivated neurons. Average firing \pm SE during the 30 -s pre- $S^{D}$ period is shown for each of the transient subtypes of Task-Activated neurons for the Early and the Late session.

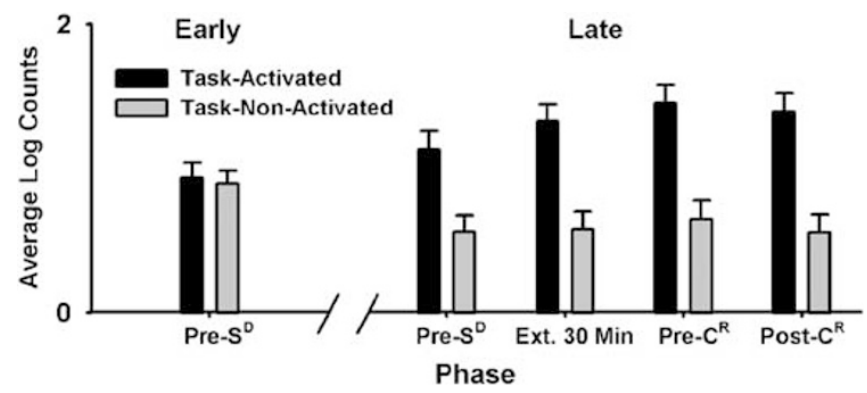

Figure 9 Average firing of Task-Activated and Task-Non-Activated neurons: No effect of the extinction and reinstatement procedure. Average firing of Task-Activated and Task-Non-Activated neurons is plotted during the following periods: (I) 30 s pre- $S^{D}$ during the Early session, (2) 30 s pre$S^{D}$ during the Late session, (3) 29.5-30 min of the extinction procedure, (4) the $30 \mathrm{~s}$ pre- $C^{R}$, and (5) the $30 \mathrm{~s}$ post- $C^{R}$.

Task-Activated and Task-Non-Activated neurons is consistent with the interpretation that the two groups of neurons showed a differential change in overall firing rates. This interpretation was supported by the findings of a number of additional analyses. The original observation of a differential between-session change in firing of Task-Activated and Task-Non-Activated neurons was replicated when comparisons were made of firing rate during longer (ie $20 \mathrm{~min}$ ) pre- and post-session baseline periods (Figure 6 and Supplementary Materials). Additionally, during the Late session, the difference in firing rate observed between the Task-Activated and Task-Non-Activated neurons was not disrupted by the response elimination and cue reinstatement procedure that followed the self-administration session (Figure 9 and Supplementary Materials). These findings show that observation of the differential betweensession change in firing did not depend on sampling firing rates during the few seconds that bracketed task events. Moreover, the between-session difference in firing was apparent during a post-session period in which expectations related to the instrumental session are expected to have been quite different relative to periods that either preceded or coincided with the self-administration session. Together, the findings are consistent with the interpretation that the differential between-session change in firing reflected a differential change in basal firing that shifted the activity of neurons correspondingly across a diverse range of behavioral conditions. The findings, in conjunction with the absence of any between-session changes in phasic firing, are also consistent with the interpretation that there were no differential changes in the event and non-event related activity of Task-Activated neurons.

Effect of subterritory. The original comparison of the TaskActivated and Task-Non-Activated groups was repeated with core $v s$ shell subterritories (defined as per Jongen-Rêlo et al, 1994) added as an additional factor. The ANOVA showed that there was a significant interaction between activation, session, and subterritory $(\mathrm{F}(1,109)=3.80, p<0.05)$. Contrasts indicated that the differential between-session change in firing of Task-Activated and Task-Non-Activated neurons was greater for the shell neurons than for the core neurons. However, when the comparison of Task-Activated vs Task-Non-Activated neurons was repeated separately 


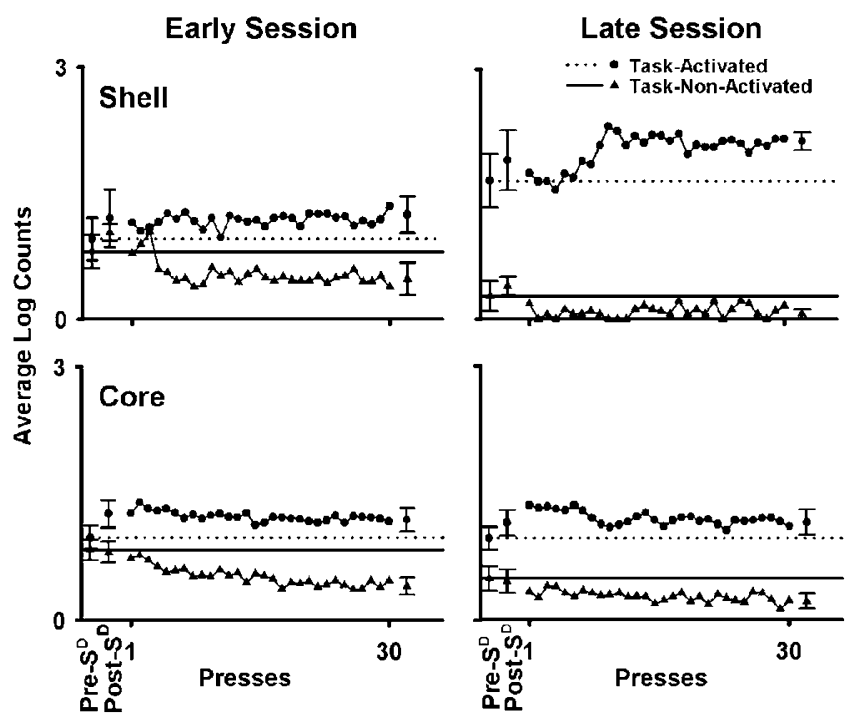

Figure 10 Average firing of Task-Activated and Task-Non-Activated neurons during the Early and the Late session: comparison between core and shell. Average firing of the Task-Activated and Task-Non-Activated neurons during various periods of the self-administration session is plotted for the Early (left column) and the Late (right column) session. Average firing rates are shown separately for shell (top) and core (bottom) neurons. From left to right on the abscissa of each plot, average firing is shown for the following periods: (I) $30 \mathrm{~s}$ pre- $S^{D},(2) 30 \mathrm{~s}$ post- $S^{\mathrm{D}}$, and (3) $30 \mathrm{~s}$ prepress for each of the first 30 cocaine-reinforced lever presses. For pre- $S^{D}$ and post- $S^{D}$, the standard error of the mean is also presented. To the immediate right of each of the plots of firing is shown average median prepress firing ( $\pm \mathrm{SE})$. Average pre- $\mathrm{S}^{\mathrm{D}}$ firing rates are shown as horizontal lines, as well as points on the graph, to facilitate between- and within-session comparisons.

for the core and the shell neurons, there was a significant interaction between activation and session for both the core neurons $(\mathrm{F}(1,86)=7.05, p<0.05)$ and the shell neurons $(\mathrm{F}(1,19)=31.13, p<0.001) \quad$ (Figure 10). Thus, Task-Activated and Task-Non-Activated neurons within both the core and the shell showed a significant differential betweensession change in firing (Figure 10).

\section{Relationship between Cocaine-Directed Behaviors and Firing of Task-Activated and Task-Non-Activated Neurons}

Behavior of high and low-moderate cocaine seekers. High cocaine seekers made a significantly greater number of lever presses during both the extinction $(\mathrm{F}(1,19)=4.86, p<0.05)$ and cue reinstatement $(\mathrm{F}(1,19)=6.26, p<0.03)$ phases than did the low-moderate cocaine seekers (Figure 11). The high cocaine-seekers also showed greater rates of cocaine taking during the Late session. Specifically, the high cocaine seekers showed a significantly lower average inter-infusioninterval during both the loading phase (ie during the first 10 presses $)(\mathrm{F}(1,19)=9.12, p=0.007)$ and the maintenance phase (ie during presses $20-30)(\mathrm{F}(1,19)=15.36, p<0.001)$ of the self-administration session (Figure 11).

Activity of neurons recorded in high vs low-moderate cocaine seekers. The difference between the firing rates of
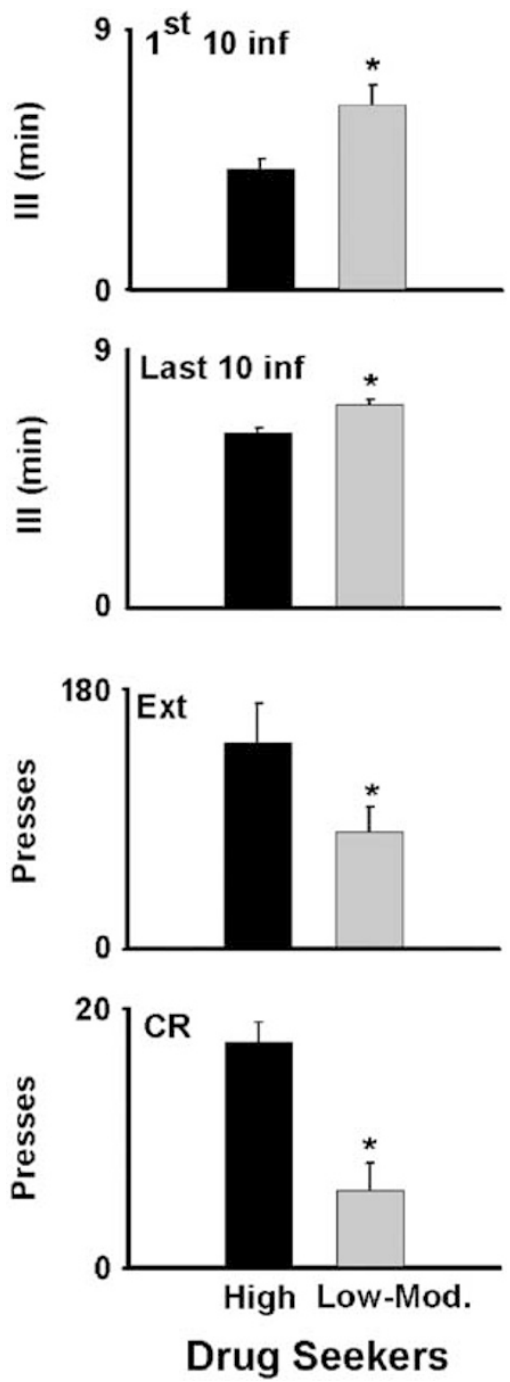

Figure I I Various indices of drug seeking and taking during the Late session are compared between the high and the low-moderate drug seekers. The bar graphs show average measures $( \pm S E)$ of the drug seeking and taking behavior exhibited by neurons that were identified as high drug seekers and low-moderate drug seekers. These measures include the following (from top to bottom of figure): (I) inter-infusion-interval (III) for the first 10 presses of the session, (2) interinfusion-interval for presses 20-30, (3) total number of presses during the extinction phase (Ext), and (4) total number of presses during the cue reinstatement phase $\left(C^{R}\right) . * P<0.05$.

Task-Activated and Task-Non-Activated neurons during various phases of the Late session were compared between the high and low-moderate cocaine seekers. An ANOVA showed that there was no significant effect of either group (high $v s$ low-moderate cocaine seekers) $(\mathrm{F}(1,51)<1.0)$ or session phase $(\mathrm{F}(7,51)=2.18, p>0.05)$ on average firing. There was also no significant interaction between either activation and session phase $(F(7,51)=2.04, p>0.05)$, session phase and group $(\mathrm{F}(7,51)<1.0)$, or activation, session phase and group $(F(7,51)<1.0)$. However, there was a significant interaction between activation and cocaine seekers $(F(7,51)=6.22, \quad p<0.02)$. Further comparisons showed that the difference between the average firing of Task-Activated and Task-Non-Activated neurons was 

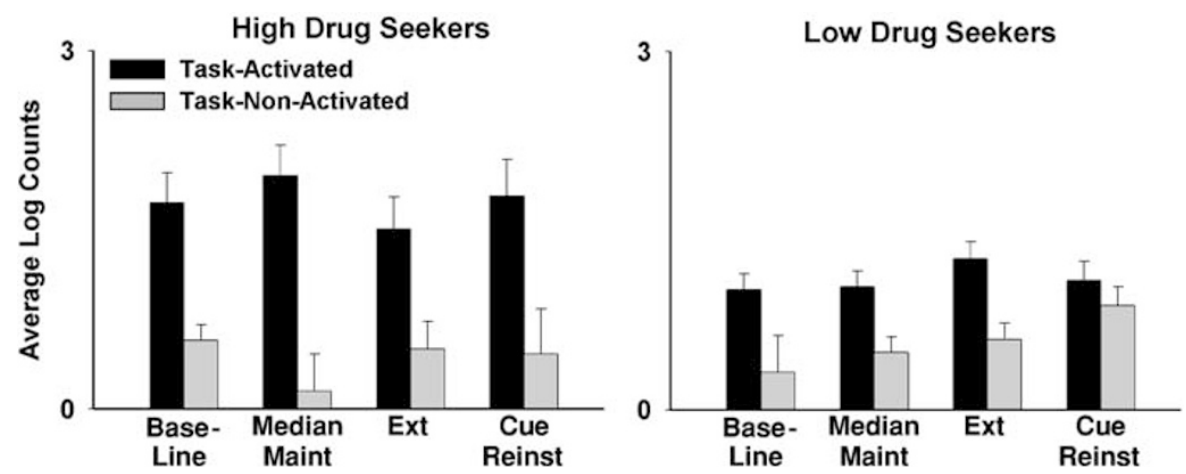

Figure 12 Average firing rates of Task-Activated and Task-Non-Activated neurons are compared between the high drug-seekers and the low-moderate drug seekers. Average firing of Task-Activated and Task-Non-Activated neurons is plotted separately for animals that were defined as high (left panel) and low-moderate (right panel) drug seekers. For each panel, average firing rates ( \pm s.e.) are shown for the following periods (top to bottom): (I) baseline (ie 30 s pre- $S^{D}$ ), (2) maintenance median pre-press period, (3) min 29.5-30 of extinction, and (4) 30 s post- $C^{R}$.

greater for the high cocaine-seekers (difference in $\log _{10}$ counts $=0.62)$ than for the low-moderate cocaine-seekers (difference in $\log _{10}$ counts $=0.36, p<0.02$ ) (Figure 12).

\section{DISCUSSION}

\section{Major Findings}

Between the Early and the Late recording session, TaskNon-Activated neurons exhibited a significant decrease in average firing rates, whereas, Task-Activated neurons did not. This differential between-session change appeared to correspond to an overall change in basal firing rate that correspondingly shifted the average firing rate of the neurons across diverse behavioral conditions. Control analyses ruled out a number of non-pharmacological factors as possible determinants of the differential between-session change in firing. The differential between-session change was greater for the shell than for the core, although, it was observed in both subterritories. The differential change in firing was associated with the emergence of a significant difference in firing between the two groups of neurons, and an increase in the ratio of the average firing rate of Task-Activated neurons relative to Task-Non-Activated neurons. The differential between-session change in firing occurred in conjunction with a significant increase in animals' rate of drug intake between the Early and the Late session. Moreover, during the Late session, the magnitude of the difference in firing rate between Task-Activated and Task-Non-Activated neurons was greater in a group of high cocaine seekers than in a group of low-moderate cocaine seekers.

\section{The Role of Learning-Related Neuroadaptations in Mediating the Differential between-Session Changes in Firing}

Given the role of accumbens in reward-related learning (Cardinal et al, 2002 for a review), it is reasonable to speculate that the differential between-session change in accumbal firing is a normal accumbal response to repeated exposure to instrumental conditioning sessions. However, this interpretation is inconsistent with a number of observations made in the present study.
Thus far, learning-related changes in striatal firing have been reported to involve changes in prevalence and amplitude of specific phasic task-related firing patterns, and to occur rapidly in response to experimental manipulations of stimulus and response contingencies (eg Aosaki et al, 1994; Kawagoe et al, 1998; Tremblay et al, 1998; Schultz et al, 2003). In contrast, no changes in magnitude and prevalence of phasic firing were observed between the Early and the Late session of the present study. Moreover, the differential change in average firing of the TaskActivated and Task-Non-Activated neurons was apparent across a diverse range of behavioral conditions (ie before, during, and after the self-administration session). Finally, the difference in firing between Task-Activated and Task-Non-Activated neurons during the Late session was insensitive to manipulations of the cue and response contingencies (ie the extinction and cue reinstatement procedure). These observations are not consistent with the interpretation that the differential between-session change in firing of the Task-Activated and Task-Non-Activated neurons corresponds to a normal accumbal response to repeated exposure to conditioning sessions. An alternative explanation of the differential change in firing is that it reflects the differential occurrence of drug-induced neuroadaptations.

\section{The Hypothesized Role of Accumbal Hypoactivity in Cocaine Addiction}

Evidence from both electrophysiological and neuro-imaging studies is consistent with the interpretation that repeated cocaine exposure leads to hypoactivity within the accumbens and larger cortico-striato-thalamo-cortical circuit. This hypoactivity has been proposed to contribute to cocaine addiction (eg Jentsch and Taylor, 1999; Volkow and Fowler, 2000; Zhang et al, 1998). In the present study, Task-Non-Activated neurons, which corresponded to approximately half of all recorded neurons, showed a significant decrease in firing between the Early and the Late recording session. Importantly, this decrease in activity was observed in wakeful animals exposed to a long-access cocaine self-administration regimen that increases drug seeking and taking (Ahmed and Koob, 1998; Paterson and Markou, 2003; Sutton et al, 2000; Ahmed and Cador, 2005). 
This finding further supports the proposal that hypoactivity in accumbens, and interconnected circuits, is induced by repeated self-administration of cocaine and contributes to cocaine addiction.

The differential neuroplasticity and differential inhibition hypotheses. Despite the evidence linking hypoactivity to cocaine addiction, the mechanisms by which hypoactivity might contribute to the differential changes in behavior that define addiction are not yet understood. Based on previous observations regarding accumbal function and neuroplasticity, we have proposed a potential mechanism. Pennartz et al (1994) proposed that the accumbal contribution to motivated behavior is mediated by differential changes in the activity of subsets, or ensembles, of neurons. Moreover, although some neurons might contribute to multiple behaviors, the particular subset that mediates any individual behavior may be unique. The make-up and genesis of ensembles of neurons that respond in a particular situation are unknown; however, the ensembles are hypothesized to consist of neurons defined by membership within particular cortico-striato-thalamo-cortical loops. Activation of particular accumbal ensembles is expected to lead to the activation of functionally related groups of neurons in downstream mesencephalic, cortical, and hypothalamic targets and to thereby contribute to the control of behavior (Pennartz et al, 1994). Consistent with this ensemble perspective, there is evidence of a dissociation between accumbal neurons that are activated during drug-directed behavior and neurons that are activated during food- or fluid-directed behaviors (Bowman et al, 1996; Carelli, 2002; Deadwyler et al, 2004). Given these observations, and evidence that neurons in the accumbens, and other components of the cortico-striato-thalamo-cortical circuit, are subject to activity-dependent plasticity (see below), we have hypothesized that differences in activity during acute drug exposure, lead to a differential occurrence of drug-induced neuroplasticity in the ensembles of synapses and neurons that are differentially involved in facilitating drug-directed behavior and that this differential neuroplasticity contributes to the differential changes in motivated behaviors that define addiction (referred to as the differential neuroplasticity hypothesis, see Peoples and Cavanaugh, 2003; Peoples et al, 2004, 2005; also see Berke and Hyman, 2000).

With respect to cocaine-induced hypoactivity in the accumbens, we hypothesize that the ensembles that contribute to drug-directed behavior are less susceptible, relative to ensembles that contribute to other motivated behaviors, to the cocaine-induced neuroadapatations that underlie hypoactivity (referred to as the differential inhibition hypothesis). A relative sparing of ensembles involved in drug seeking and taking from the inhibitory effects of repeated drug exposure would be associated with a relative increase in the transmission of signals that facilitate drug-directed behavior through accumbal circuits, and an absolute decline in the transmission of signals that promote alternative motivated behaviors. This increase in selective throughput would be amplified even further if TaskActivated neurons underwent alternative excitatory adaptations. A narrowing of information flow through accumbal circuits could contribute to the selective increase in drug seeking and drug taking and the simultaneous decline in other behaviors, essentially locking an individual into an uncontrollable, involuntary, pattern of drug seeking and taking. Given the involvement of accumbens, and interconnected structures in a variety of behaviors, motivated by appetitive as well as aversive outcomes, the differential hypoactivity in neurons other than those that contribute to drug seeking and taking could also contribute to negative symptoms of addiction such as anhedonia, and the loss of inhibitory control by adverse behavioral outcomes (Peoples, 2002; Peoples et al, 2004, 2005; also see Volkow et al, 2002; Zang et al, 1998).

Predictions and evidence related to the differential neuroplasticity and differential inhibition hypotheses. There are numerous predictions that one would expect to hold true if the differential inhibition hypothesis were correct. One basic prediction is that neurons that are activated during the self-administration task will show less evidence of a between-session decrease in basal firing than will other neurons. Consistent with this prediction, firing rate during drug-free baseline periods decreased for TaskNon-Activated neurons but not for Task-Activated neurons. Importantly, the differential between-session change in baseline firing was associated with comparable changes in firing across diverse behavioral conditions. This finding is consistent with the interpretation that the change in firing might reflect a differential change in overall excitability of the neurons, as would be consistent with findings of acute recording studies of cocaine-induced hypoactivity (eg Zhang et al, 1998, 2002). An important implication of this type of plasticity is that the changes in firing have the potential to influence drug-directed behavior across a broad range of conditions. The degree to which the changes in firing really impact motivated behavior directed toward drug and non-drug rewards remains to be determined. However, in the present study, the firing rate changes co-varied with the propensity of animals to take cocaine. Specifically, the differential between-session change in firing was associated with an increase in the average rate of drug intake between the Early and the Late session. Moreover, during the Late session, the magnitude of the difference in firing between Task-Activated and Task-NonActivated neurons was positively related to the propensity of animals to engage in drug-directed behavior. Although further investigation of the findings of the present study are required, the data are consistent with the hypothesis that neurons that are activated during cocaine self-administration are less susceptible to cocaine-induced hypoactivity relative to other neurons. Additionally, differential hypoactivity between neurons that do and do not facilitate drugdirected behavior might contribute to the differential changes in behavior that define cocaine addiction.

In addition to differential plasticity between neurons, the differential neuroplasticity and inhibition hypotheses posit that differential plasticity might occur among synapses that are differentially activated during periods of drug exposure (Berke and Hyman, 2000; Peoples and Cavanaugh, 2003). Transiently Task-Activated neurons (ie phasically activated neurons) show increased rates of firing during specific task-related periods relative to other periods in the session. These differences in firing rate likely correspond to 
differential changes in activity across synapses of the individual neurons. This differential synaptic activity could lead to differential plasticity such that firing of the TaskActivated neurons during task events might be enhanced relative to firing during other periods of the session. This enhancement of phasic activity could involve either an increase in firing rate during task-event intervals (ie an increase in signal firing), a decrease in firing during nontask-event intervals (ie a decrease in background firing), or both. There was no evidence in the present study of such changes in firing of individual Task-Activated neurons (ie Table 3).

However, in the study of Hollander and Carelli (2005), there was evidence of such an enhancement in phasic firing. It is possible that such differential within-neuron changes in firing depend on neuroadaptations that occurred in the previous study but not in the present study. This proposal is plausible given that cocaine-induced neuroadaptations depend on a number of pharmacological parameters that differed between the two experiments. Perhaps most important, the animals in the previous study were exposed to more limited drug exposure and a longer period of drug abstinence than were animals of the present study. One implication of this interpretation is that the within-neuron changes in firing might be relevant to some but not other stages of the natural history of drug addiction (eg preaddiction drug use, maintenance of drug addiction, and relapse). The same could also be true for the differential between-neuron decrease in firing observed in the present study.

\section{Core vs Shell}

In the present experiment, a differential between-session decrease in average firing was exhibited by both shell and core neurons, although the magnitude of the change was greater for the former than for the latter. The presence of significant differential changes in firing of Task-Activated and Task-Non-Activated neurons in both the core and the shell is consistent with the interpretation that differential hypoactivity is potentially important in mediating the influence of each subterritory to drug-directed behavior. However, the core and the shell modulate different aspects of drug-directed behavior. For example, the shell appears to be more important than the core to the reinforcing properties of cocaine and other psychomotor stimulants (McBride et al, 1999; Bari and Pierce, 2005; Ikemoto et al, 2005; also see Ito et al, 2004). In contrast, the core appears to be more important than the shell to the incentive motivational properties of drug and drug-associated cues (McFarland and Kalivas, 2001; Kalivas and McFarland, 2003; McFarland et al, 2003; Fuchs et al, 2004; also see Fuchs et al, 2006). Differential hypoactivity in the two subterritories might therefore be expected to contribute differently to changes in behavior that occur with repeated exposure to cocaine self-administration.

The core and the shell differ in anatomical, neurochemical and electrophysiological characteristics (eg Pennartz et al, 1992; Jongen-Rêlo et al, 1994; Heimer et al, 1997; Groenewegen et al, 1999; Zahm, 2000). Moreover, subterritorial differences in cocaine-induced neuroadaptations have been observed in previous studies (eg Sorg et al, 1995;
Robinson et al, 2001; Thomas et al, 2001; Ricci et al, 2004; Brenhouse and Stellar, 2006). The different magnitude of change in firing between core and shell in the present study might thus correspond to a subterritorial difference in the types of neuroadaptations that are induced by cocaine. However, there is evidence that cocaine-induced adaptations can progress from more medial and ventral portions of striatum, to more lateral and dorsal regions (Porrino et al, 2004). It is thus possible that the greater magnitude change in shell relative to core reflects shared neuroadaptation(s) that occur more rapidly, or with less drug exposure, in the shell relative to the core. Finally, it is possible that the firing rate changes in the core reflected an influence of the shell on activity of core neurons via stiato-thalamo-corticostriato connections (cf, Zahm, 2000).

\section{Cocaine-Induced Neuroadaptations that Potentially Mediated the Differential between-Session Changes in Firing of Task-Activated and Task-Non-Activated Neurons}

Repeated exposure to cocaine leads to lasting adaptations in the mesoaccumbal DA pathway, although the nature of these adaptations depend on drug dose, inter-exposureinterval, duration of exposure, and period of withdrawal. In general, limited, intermittent, drug exposure leads to a facilitation of cocaine-induced increases in accumbal DA, an augmentation of evoked DA release, and an upregulation of $\mathrm{D}_{1}$ DA receptor function. These adaptations are most consistently observed at late withdrawal time points (Pierce and Kalivas, 1997; Vanderschuren and Kalivas, 2000, for a review). The same regimens do not appear to have a consistent effect on basal DA levels (Chefer and Shippenberg, 2002; Licata and Pierce, 2004, for a review).

A shorter withdrawal period, combined with more extensive drug exposure, can lead to decreases in measures of accumbal DA function, including decreased DA, and decreased $D_{1}$ and $D_{2}$ receptor function (eg Hammer et al, 1997; Nader et al, 2002; Self, 2004; Mateo et al, 2005). This latter set of conditions is most consistent with the drug exposure of the present study. It is therefore more likely that accumbal DA activity underwent a downregulation rather than an upregulation between the Early and the Late recording session. Consistent with this speculation, Ahmed and co-workers documented changes in dopaminergic neurotransmission in animals exposed to the 30-day longaccess cocaine self-administration regimen. The drug exposure did not alter the potency of cocaine to increase accumbal DA levels. However, it increased the sensitivity of animals to antagonism of cocaine reinforcement by a nonselective $\mathrm{D}_{1} / \mathrm{D}_{2}$ antagonist (Ahmed et al, 2003; Ahmed and Koob, 1998). It is possible that this and other changes in dopaminergic transmission occurred differentially at pre- and postsynaptic terminals of Task-Activated and Task-Non-Activated neurons. However, it is also possible that alternative differential neuroadaptations influenced the response of those subsets of neurons to dopaminergic adaptations.

Consistent with this latter possibility, the impact of repeated cocaine exposure on the accumbens is not limited to changes in DA transmission. For example, repeated exposure to cocaine can lead to decreased basal presynaptic 
and extracellular glutamate levels in the accumbens (Keys et al, 1998; Meshul et al, 1998; Bell et al, 2000; Hotsenpiller et al, 2001; Baker et al, 2003), increased drug-evoked glutamate release (Pierce et al, 1996), and changes in glutamate receptors ( $\mathrm{Lu}$ et al, 2003; Tang et al, 2004; Boudreau and Wolf, 2005). A history of cocaine exposure can also be associated with changes in accumbal levels of other neurochemicals (Parsons and Justice, 1993; Xi et al, 2003), growth factors (Grimm et al, 2003), and receptors (Przegaliñski et al, 2003; Ricci et al, 2004) as well as evidence of postsynaptic changes in signal transduction, gene transcription factors (McClung and Nestler, 2003; Nestler, 2005) and cell morphology (Robinson et al, 2001; Ferrario et al, 2005; Norrholm et al, 2003). Most of these pre- and postsynaptic neuroadaptations have been characterized under experimental conditions that are quite different from those of the present study. However, certain are expected to be inhibitory (eg Hu et al, 2005; McClung and Nestler, 2003; Nestler, 2005). Others are expected to be excitatory (Pierce et al, 1996; Grimm et al, 2003; Boudreau and Wolf, 2005; McClung and Nestler, 2003; Ferrario et al, 2005). It is possible that some of the 'excitatory' and 'inhibitory' adaptations occurred differentially in the TaskActivated and Task-Non-Activated neurons respectively. This proposal is consistent with the suggestion that some of the excitatory neuroadaptations are compensatory responses that limit cocaine-induced hypoactivity (Boudreau and Wolf, 2005; Bibb et al, 2001; Dong et al, 2006).

We have hypothesized that differential pre- and postsynaptic neuroadaptations might be mediated by differences in activity during periods of drug exposure (see above). Differences in activity during drug exposure could be associated with differential neurochemical, neurophysiological and molecular events and influence the occurrence of multiple types of drug-induced plasticity. For example, the differences in activity between TaskActivated and Task-Non-Activated neurons would be expected to be associated with a differential influx of $\mathrm{Ca}^{2+}$, which could influence a number of $\mathrm{Ca}^{2+}$ mediated cocaine-induced neuroadaptations, including those that are hypothesized to contribute to cocaine-induced hypoactivity of accumbal neurons (see Hu et al, 2005; Valjent et al, 2005; Dong et al, 2006).

We have also proposed that DA may play an important role in activity-dependent neuroadaptations (Peoples and Cavanaugh, 2003; Peoples et al, 2004). Cocaine, like other addictive drugs, acutely elevates accumbal DA (Di Chiara, 1998 for a review). The acute effects of DA on target neurons appears to vary depending on the activity of accumbal neurons at the time of DA exposure: It facilitates depolarizing currents in neurons that are receiving robust excitatory input and inhibits those currents in neurons that are receiving weak or no excitatory input (Nicola et al, 2000, for a review). The different excitatory afferent input impinging on Task-Activated and Task-Non-Activated neurons during the period of drug exposure would thus be expected to facilitate firing of Task-Activated neurons and to inhibit the activity of Task-Non-Activated neurons (eg Rolls et al, 1984; DeFrance et al, 1985; Pennartz et al, 1994; Kiyatkin and Rebec, 1996; Nicola et al, 2000; O'Donnell, 2003). Consistent with this expectation, TaskActivated neurons, on average, achieve and maintain higher average firing rates than do Task-Non-Activated neurons during periods of cocaine self-administration (Peoples et al, 2004). Activity-dependent acute effects of DA could make the Task-Activated neurons less susceptible to DA-mediated mechanisms that contribute to hypoactivity (for examples of such mechanisms see Zhang et al, 1998, 2002; Hu et al, 2005; also see Dong et al, 2006) and potentially more susceptible to various types of neuroadaptations that are expected to have an excitatory impact on synaptic and neuronal activity (see above).

The findings of the present study are consistent with a possible contribution of activity-dependent plasticity to differential cocaine-induced neuroadaptations. In particular, the analysis of the session-increase, Task-But-NotSession-Activated, and Task-Non-Activated neurons suggests that between-session change in firing exhibited by neurons is positively related to the type of within-session change in firing exhibited by neurons during individual cocaine self-administration sessions. Session-increase neurons maintained elevated rates of firing throughout individual self-administration sessions and showed a trend to increase baseline firing rates between the Early and the Late sessions. The majority of Task-But-Not-Session-Activated neurons (76\%) were session-decrease neurons. However, firing of the neurons, on average, transiently approximated baseline firing rates around the time of each cocainereinforced lever press (eg Figure 5). These neurons showed no between-session change in baseline firing rates. Finally, the majority of Task-Non-Activated neurons ( $>75 \%)$ showed a consistent suppression in firing throughout individual self-administration sessions and the group, as a whole, showed a between-session decrease in basal firing rates.

Data of other laboratories show that accumbal neurons undergo activity-dependent plasticity (eg Pennartz et al, 1993, 1994; Kombian and Malenka, 1994) and that certain cocaine-induced neuroaptations are activity-dependent (Thomas et al, 2000; Valjent et al, 2005). A specific role for activity-dependent plasticity in mediating the differential between-session change in firing observed in the present experiment, and a more general role of activity-dependent plasticity in mediating differential cocaine-induced neuroadaptations, thus seems plausible. An interesting implication of the hypothesis is that once differential neuroadaptations have occurred, the resulting disparity in neuronal activity could facilitate further differential adaptations that could either maintain or increase the relative difference in signaling strength. Such a process would be consistent with the progressive and chronic nature of drug addiction. Further studies of the influence of physiologically relevant variations in neuronal activity on mechanisms that mediate cocaine-induced neuroadaptations may provide novel insight into mechanisms that contribute to cocaine addiction.

\section{ACKNOWLEDGEMENTS}

Jason Collison, Alexis Simpson, and Jillian O'Toole contributed to data analysis and figure construction. David E Moorman provided helpful comments on the manuscript. Anthony J Uzwiak and Anthony T Fabbricatore contributed to data collection. Linda King contributed to the histological 
procedures. Research funded by DA13401 (LL Peoples), DA06886 (MO West), and DA05186 (CP O'Brien).

\section{REFERENCES}

Ahmed SH, Cador M (2005). Dissociation of psychomotor sensitization from compulsive cocaine consumption. Neuropsychopharmacology 31: 563-571.

Ahmed SH, Koob GF (1998). Transition from moderate to excessive drug intake: change in hedonic set point. Science 282: 298-300.

Ahmed SH, Lin D, Koob GF, Parsons LH (2003). Escalation of cocaine self-administration does not depend on altered cocaineinduced nucleus accumbens dopamine levels. J Neurochem 86: 102-113.

American Psychiatric Association (1994). Diagnostic and Statistical Manual of Mental Disorders, 4th edn. American Psychiatric Association: Washington, DC.

Aosaki T, Tsubokawa H, Ishida A, Watanabe K, Graybiel AM, Kimura M (1994). Responses of tonically active neurons in the primate's striatum undergo systematic changes during behavioral sensorimotor conditioning. J Neurosci 14: 3969-3984.

Baker DA, McFarland K, Lake RW, Shen H, Toda S, Kalivas PW (2003). Neuroadaptations in cystine-glutamate exchange underlie cocaine relapse. Nat Neurosci 6: 743-749.

Bari AA, Pierce RC (2005). D1-like and D2 dopamine receptor antagonists adminsiterd into the shell subregion of the rat nucleus accumbens decrease cocaine, but not food, reinforcement. Neuroscience 135: 959-968.

Bell K, Duffy P, Kalivas PW (2000). Context-specific enhancement of glutamate transmission by cocaine. Neuropsychopharmacology 23: 335-344.

Berke JD, Hyman SE (2000). Addiction, dopamine, and the molecular mechanisms of memory. Neuron 25: 515-532.

Beurrier C, Malenka RC (2002). Enhanced inhibition of synaptic transmission by dopamine in the nucleus accumbens during behavioral sensitization to cocaine. J Neurosci 22: 5817-5822.

Bibb JA, Chen J, Taylor JR, Svenningsson P, Nishi A, Snyder GL et al (2001). Effects of chronic exposure to cocaine are regulated by the neuronal protein Cdk5. Nature 410: 376-380.

Blackburn JR, Pfaus JG, Phillips AG (1992). Dopamine functions in appetitive and defensive behaviors. Prog Neurobiol 39: 247-279.

Boudreau AC, Wolf ME (2005). Behavioral sensitization to cocaine is associated with increased AMPA receptor surface expression in the nucleus accumbens. J Neurosci 25: 9144-9151.

Bowman EM, Aigner TG, Richmond BJ (1996). Neural signals in the monkey ventral striatum related to motivation for juice and cocaine rewards. J Neurophysiol 75: 1061-1073.

Brenhouse HC, Stellar JR (2006). c-Fos and deltaFosB expression are differentially altered in distinct subregions of the nucleus accumbens shell in cocaine-sensitized rats. Neuroscience 137: 773-780.

Cardinal RN, Parkinson JA, Hall J, Everitt BJ (2002). Emotion and motivation: the role of the amygdale, ventral striatum, and prefrontal cortex. Neurosci Biobehav Rev 26: 321-352.

Carelli RM (2002). Nucleus accumbens cell firing during goaldirected behaviors for cocaine vs 'natural' reinforcement. Physiol Behav 76: 379-387.

Carelli RM, King VC, Hampson RE, Deadwyler SA (1993). Firing patterns of nucleus accumbens neurons during cocaine selfadministration in rats. Brain Res 626: 14-22.

Chang J-Y, Sawyer SF, Lee RS, Woodward DJ (1994). Electrophysiological and pharmacological evidence for the role of the nucleus accumbens in cocaine self-administration in freely moving rats. J Neurosci 14: 1224-1244.

Chefer VI, Shippenberg TS (2002). Changes in basal and cocaineevoked extracellular dopamine uptake and release in the rat nucleus accumbens during early abstinence from cocaine: quantitative determination under transient conditions. Neuroscience 112: 907-919.

Deadwyler SA, Hayashizaki S, Cheer J, Hampson RE (2004). Reward, memory and substance abuse: functional neuronal circuits in the nucleus accumbens. Neurosci Biobehav Rev 27: 703-711.

DeFrance JF, Sikes RW, Chronister RB (1985). Dopamine action in the nucleus accumbens. J Neurophysiol 54: 1568-1577.

Deroche-Gamonet V, Belin D, Piazza PV (2004). Evidence for addiction-like behavior in the rat. Science 305: 1014-1017.

deWit H, Stewart J (1981). Reinstatement of cocaine-reinforced responding in the rat. Psychopharmacology 75: 134-143.

Di Chiara G (1998). A motivational learning hypothesis of the role of mesolimbic dopamine compulsive drug uses. J Psychopharmacol 12: 54-67.

Dong Y, Green T, Saal D, Marie H, Neve R, Nestler EJ et al (2006). CREB modulates excitability of nucleus accumbens neurons. Nat Neurosci 9: 475-477.

Everitt BJ (1990). Sexual motivation: a neural and behavioral analysis of the mechanisms underlying appetitive and copulatory responses of male rats. Neurosci Biobehav Rev 14: 217-232.

Febo M, Segarra AC, Nair G, Schmidt K, Duong TQ, Ferris CF (2005). The neural consequences of repeated cocaine exposure revealed by functional MRI in awake rats. Neuropsychopharmacology 30: 936-943.

Ferrario CR, Grazyna G, Crombag HS, Li Y, Kolb B, Robinson TE (2005). Neural and behavioral plasticity associated with the transition from controlled to escalated cocaine use. Biol Psych 58: 751-759.

Fuchs RA, Branham RK, See RE (2006). Different neural substrates mediate cocaine seeking after abstinence versus extinction training: a critical role for the dorsolateral caudate-putamen. J Neurosci 26: 3584-3588.

Fuchs RA, Evans KA, Parker MC, See R (2004). Differential involvement of the core and shell subregions of the nucleus accumbens in conditioned cue-induced reinstatement of cocaine seeking in rats. Psychopharmacology 176: 459-465.

Green JD (1958). A simple microelectrode for recording from the central nervous system. Nature 182: 962.

Grimm JW, Lu L, Hayashi T, Hope BT, Su T-P, Shaham Y (2003). Time-dependent increases in brain-derived neurotrophic factor protein levels within the mesolimbic dopamine system after withdrawal from cocaine: implications for incubation of cocaine craving. J Neurosci 23: 742-747.

Groenewegen HJ, Wright C, Beijer AVJ, Voorn P (1999). Convergence and segregation of ventral stratal inputs and outputs. Ann NY Acad Sci 877: 49-63.

Hammer Jr RP, Egilmez Y, Emmett-Oglesby MW (1997). Neural mechanisms of tolerance to the effects of cocaine. Behav Brain Res 84: 225-239.

Heimer L, Alheid GF, de Olmos J, Groenewegen HJ, Haber SN, Harlan SN et al (1997). The accumbens: beyond the core-shell dichotomy. J Neuropsychiatry Clin Neurosci 9: 354-381.

Hollander JA, Carelli RM (2005). Abstinence from cocaine selfadministration heightens neural encoding of goal-directed behaviors in the accumbens. Neuropsychopharmacology 30: 1464-1474.

Hotsenpiller G, Giorgetti M, Wolf ME (2001). Alterations in behavior and glutamate transmission following presentation of stimuli previously associated with cocaine exposure. Eur $J$ Neurosci 14: 1843-1855.

Hu X-T, Ford K, White FJ (2005). Repeated cocaine administration decreases calcineurin (PP2B) but enhances DARPP-32 modulation of sodium currents in rat nucleus accumbens neurons. Neuropsychopharmacology 30: 916-926.

Ikemoto S, Panksepp J (1999). The role of nucleus accumbens dopamine in motivated behavior: a unifying interpretation with special reference to reard-seeking. Brain Res Rev 31: 6-41. 
Ikemoto S, Qin M, Liu Z-H (2005). The functional divide for primary reinforcement of d-amphetamine lies between the medial and lateral ventral striatum: is the division of the accumbens core, shell, and olfactory tubercle valid? J Neurosci 25: 5061-5065.

Ito R, Robbins TW, Everitt BJ (2004). Differential control over cocaine-seeking behavior by nucleus accumbens core and shell. Nat Neurosci 7: 389-397.

Jentsch JD, Taylor JR (1999). Impulsivity resulting from frontostriatal dysfunction in drug abuse: implications for the control of behavior by reward-related stimuli. Psychopharmacology 146: 373-390.

Jongen-Rêlo AL, Voorn P, Groenewegen HJ (1994). Immunohistochemical characterization of the shell and core territories of the nucleus accumbens in the rat. Eur J Neurosci 6: 1255-1264.

Kalivas PW, McFarland K (2003). Brain circuitry and the reinstatement of cocaine-seeking behavior. Psychopharmacology 168: $44-56$.

Kaufman JN, Ross TJ, Stein EA, Garavan H (2003). Cingulate hypoactivity in cocaine users during a GO-NOGO task as revealed by event-related functional magnetic resonance imaging. J Neurosci 23: 7839-7843.

Kawagoe R, Takikawa Y, Hikosaka O (1998). Expectation of reward modulates cognitive signals in the basal ganglia. Nat Neurosci 1: 411-416.

Kelley AE (2004). Ventral striatal control of appetitive motivation: role in ingestive behavior and reward-related learning. Neurosci Biobehav Rev 27: 765-776.

Keys AS, Mark GP, Emre N, Meshul CK (1998). Reduced glutamate immunolabeling in the nucleus accumbens following extended withdrawal from self-administered cocaine. Synapse 30: 393-401.

Kiyatkin EA, Rebec GV (1996). Dopaminergic modulation of glutamate-induced excitations of neurons in neostriatum and nucleus accumbens of awake, unrestrained rats. J Neurophysiol 75: 142-153.

Kombian SB, Malenka RC (1994). Simultaneous LTP of nonNMDA- and LTD of NMDA-receptor-mediated responses in the nucleus accumbens. Nature 17: 242-246.

Licata SC, Pierce RC (2004). Repeated cocaine injections have no influence on tyrosine hydroxylase activity in the rat nucleus accumbens core or shell. Brain Res 1012: 119-126.

London ED, Bonson KR, Ernst M, Grant S (1999). Brain imaging studies of cocaine abuse: implications for medication development. Crit Rev Neurobio 13: 227-242.

Lu L, Grimm JW, Shaham Y (2003). Molecular neuroadaptations in the accumbens and ventral tegmental area during the first 90 days of forced abstinence from cocaine self-administration in rats. J Neurochem 85: 1604-1613.

Macey DJ, Rice WN, Freedland CS, Whitlow CT, Porrino LJ (2004). Patterns of functional activity associated with cocaine selfadministration in the rat change over time. Psychopharmacology 172: 384-392.

Mateo Y, Lack CM, Morgan D, Roberts DCS, Jones SR (2005). Reduced dopamine terminal function and insensitivity to cocaine following cocaine binge self-administration and deprivation. Neuropsychopharmacology 30: 1455-1463.

McBride WJ, Murphy JM, Ikemoto S (1999). Localization of brain reinforcement mechanisms: intracranial self-administration and intracranial place-conditioning studies. Behav Brain Res 101: 129-152.

McClung CA, Nestler EJ (2003). Regulation of gene expression and cocaine reward by CREB and deltaFosB. Nat Neurosci 6: 1208-1215.

McFarland K, Kalivas PW (2001). The circuitry mediating cocaineinduced reinstatement of drug-seeking behavior. J Neurosci 21: 8655-8663.

McFarland K, Lapish CC, Kalivas PW (2003). Prefrontal glutamate release into the core of the nucleus accumbens mediates cocaine- induced reinstatement of drug-seeking behavior. J Neurosci 23: 3531-3537.

Meshul CK, Noguchi K, Emre N, Ellison G (1998). Cocaine-induced changes in glutamate and GABA immunolabeling within rat habenula and nucleus accumbens. Synapse 30: 211-220.

Nader MA, Daunais JB, Moore T, Nader SH, Moore RJ, Smith HR et al (2002). Effects of cocaine self-administration on striatal dopamine systems in rhesus monkeys: initial and chronic exposure. Neuropsychopharmacology 27: 35-46.

Nestler EJ (2005). Is there a common molecular pathway for addiction? Nat Neurosci 8: 1445-1449.

Nicola SM, Surmeier J, Malenka RC (2000). Dopaminergic modulation of neuronal excitability in the striatum and nucleus accumbens. Annu Rev Neurosci 23: 185-212.

Norrholm SD, Bibb JA, Nestler EJ, Ouimet CC, Taylor JR, Greengard P (2003). Cocaine-induced proliferation of dendritic spines in nucleus accumbens is dependent on the activity of cyclin-dependent kinase-5. Neuroscience 116: 19-22.

O'Donnell P (2003). Dopamine gating of forebrain neural ensembles. J Neurosci 17: 429-435.

Pan HT, Menacherry S, Justice Jr JB (1991). Differences in the pharmacokinetics of cocaine in naïve and cocaine-experienced rats. J Neurochem 56: 1299-1306.

Parsons LH, Justice Jr JB (1993). Serotonin and dopamine sensitization in the nucleus accumbens, ventral tegmental area, and dorsal raphe nucleus following repeated cocaine administration. J Neurochem 61: 1611-1619.

Paterson NE, Markou A (2003). Increased motivation for selfadministerd cocaine after escalated cocaine intake. Neuroreport 14: 2229-2232.

Paxinos G, Watson C (1996). The Rat Brain in Stereotaxic Coordinates. Academic: San Diego, CA.

Pennartz CM, Groenewegen HJ, Lopes da Silva FH (1994). The nucleus accumbens as a complex of functionally distinct neuronal ensembles: an integration of behavioral, electrophysiological and anatomical data. Prog Neurobiol 42: 719-761.

Pennartz CMA, Ameerun RF, Groenewegen HJ, Lopes da Silva FH (1993). Synaptic plasticity in an in vitro slice preparation of the rat nucleus accumbens. Eur J Neurosci 5: 107-117.

Pennartz CMA, Dolleman-Van der Weel MJ, Lopes da Silva FH (1992). Differential membrane properties and dopamine effects in the shell and core of the rat nucleus accumbens studied in vitro. Neurosci Lett 136: 109-112.

Peoples LL (2002). Will, Anterior cingulated cortex, and addiction. Science 296: 1623-1624.

Peoples LL (2003). Application of chronic extracellular recording to studies of drug self-administration. In: Waterhouse BD, Nicolelis M (eds). Methods in Drug Abuse Research: Cellular and Circuit Level Analyses. CRC: Boca Raton, FL. pp 161-211.

Peoples LL, Cavanaugh DJ (2003). Differential changes in signal and background firing of accumbal neurons during cocaine selfadministration. J Neurophysiol 90: 993-1010.

Peoples LL, Gee F, Bibi R, West MO (1998a). Phasic firing time locked to cocaine self-infusion and locomotion: dissociable firing patterns of single nucleus accumbens neurons in the rat. J Neurosci 18: 7588-7598.

Peoples LL, Kravitz AV, Lynch KG (2005). The differential neuroplasticity and differential inhibition hypothesies of drug addiction: empirical evidence and implications for treatment. Neuropsychopharmacology 30: S34.

Peoples LL, Lynch KG, Lesnock J, Gangadhar N (2004). Accumbal neural responses during the initiation and maintenance of intravenous cocaine self-administration. J Neurophysiol 91: 314-323 (online October 2003).

Peoples LL, Uzwiak AJ, Gee F, West MO (1997). Operant behavior during sessions of intravenous cocaine infusions is necessary and sufficient for phasic firing of single nucleus accumbens neurons. Brain Res 757: 280-284. 
Peoples LL, Uzwiak AJ, Gee F, West MO (1998b). Tonic inhibition of single nucleus accumbens neurons in the rat: a predominant but not exclusive firing pattern induced by cocaine selfadministration sessions. Neuroscience 86: 13-22.

Peoples LL, Uzwiak AJ, Gee F, West MO (1999). Tonic firing of rat nucleus accumbens neurons: changes during the first 2 weeks of daily cocaine self-administration sessions. Brain Res 822: 231-236.

Peoples LL, West MO (1996). Phasic firing of single neurons in single neurons in the rat nucleus accumbens correlated with the timing of intravenous cocaine self-administration. J Neurosci 16: 3459-3473.

Pierce RC, Bell K, Duffy P, Kalivas PW (1996). Repeated cocaine augments excitatory amino acid transmission in the nucleus accumbens only in rats having developed behavioral sensitization. J Neurosci 16: 1550-1560.

Pierce RC, Kalivas PW (1997). A circuitry model of the expression of behavioral sensitization to amphetamine-like psychostimulants. Brain Res Rev 25: 192-216.

Porrino LJ, Lyons D, Miller MD, Smith HR, Friedman DP, Daunais JB et al (2002). Metabolic mapping of the effects of cocaine during the initial phases of self-administration in the nonhuman primate. J Neurosci 22: 7687-7694.

Porrino LJ, Lyons D, Smith HR, Daunais JB, Nader MA (2004). Cocaine self-administration produces a progressive involvement of limbic, association, and sensorimotor striatal domains. J Neurosci 24: 3554-3562.

Przegaliñski E, Czepiel K, Nowak E, Dlaboga D, Filip M (2003). Withdrawal from chronic cocaine up-regulates $5-\mathrm{HT}_{1 \mathrm{~B}}$ receptors in the rat brain. Neurosci Let 351: 169-172.

Ricci LA, Stellar JR, Todtenkopf MS (2004). Subregion-specific down-regulation of $5-\mathrm{HT}_{3}$ immunoreactivity in the nucleus accumbens shell during the induction of cocaine sensitization. Pharmacol Biochem Behav 77: 415-422.

Robinson TE, Gorney G, Mitton E, Kolb B (2001). Cocaine selfadministration alters the morphology of dendrites and dendritic spines in the nucleus accumbens and neocortex. Synapse 39: 257-266.

Rolls ET, Thorpe SJ, Boytim M, Szabo I, Perrett DI (1984). Responses of striatal neurons in the behaving monkey. 3. Effects of iontophoretically applied dopamine on normal responsiveness. Neuroscience 12: 1201-1212.

Salamone JD, Correa M, Mingote SM, Weber SM (2005). Beyond the reward hypothesis: alternative functions of nucleus accumbens dopamine. Curr Opin Pharmacol 5: 34-41.

Schultz W, Tremblay L, Hollerman JR (2003). Changes in behavior-related neuronal activity in the striatum during learning. Trend Neurosci 26: 321-328.

Schwienbacher I, Fendt M, Richardson R, Schnitzler H-U (2004). Temporary inactivation of the nucleus accumbens disrupts acquisition and expression of fear-potentiated startle in rats. Brain Res 1027: 87-93.

Self DW (2004). Regulation of drug-taking and -seeking behaviors by neuroadaptations in the mesolimbic dopamine system. Neuropharmacology 47: 242-255.

Sorg BA, Guminski BJM, Hooks MS, Kalivas PW (1995). Cocaine alters glutamic acid decarboxylase differentially in the nucleus accumbens core and shell. Mol Brain Res 29: 381-386.

Sutton MA, Karanian DA, Self DW (2000). Factors that determine a propensity for cocaine-seeking behavior during abstinence in rats. Neuropsychopharmacology 22: 626-641.
Tang W, Wesley M, Freeman WM, Liang B, Hemby SE (2004). Alterations in ionotropic glutamate receptor subunits during binge cocaine self-administration and withdrawal in rats. J Neurochem 89: 1021-1033.

Thomas MJ, Beurrier C, Bonci A, Malenka RC (2001). Long-term depression in the nucleus accumbens: a neural correlate of behavioral sensitization to cocaine. Nat Neurosci 4: 1217-1223.

Thomas MJ, Malenka RC, Bonci A (2000). Modulation of long-term depression by dopamine in the mesolimbic system. J Neurosci 20: 5581-5586.

Tremblay L, Hollerman JR, Schultz W (1998). Modifications of reward expectation-related neuronal activity during learning in primate striatum. J Neurophysiol 80: 964-977.

Uzwiak AJ, Guyette FX, West MO, Peoples LL (1997). Neurons in accumbens subterritor4ies of the rat: phasic firing time-locked within seconds of intravenous cocaine self-infusion. Brain Res 767: 363-369.

Valjent E, Pascoli V, Svenningsson P, Paul S, Enslen H, Corvol J-C et al (2005). Regulation of a protein phosphatase cascade allows convergent dopamine and glutamate signals to activate ERK in the striatum. Proc Natl Acad Sci USA 102: 491-496.

Vanderschuren LJ, Kalivas PW (2000). Alterations in dopaminergic and glutamatergic transmission in the induction and expression of behavioral sensitization: a critical review of preclinical studies. Psychopharmacology 151: 99-120.

Volkow ND, Fowler JS (2000). Addiction, a disease of compulsion and drive: involvement of the orbitofrontal cortex. Cereb Cortex 10: 318-325.

Volkow ND, Fowler JS, Wang G-J, Goldstein RZ (2002). Role of dopamine, the frontal cortex and memory circuits in drug addiction: insight from imaging studies. Neurobio Learn Mem 78: $610-624$.

Volkow ND, Fowler JS, Wang GJ, Hitzemann R, Logan J, Schlyer DJ et al (1993). Decreased dopamine D2 receptor availability is associated with reduced frontal metabolism in cocaine abusers. Synapse 14: 169-177.

White FJ, Hu X-T, Zhang X-F, Wolf ME (1995a). Repeated administration of cocaine or amphetamine alters neuronal responses to glutamate in the mesoaccumbens dopamine system. J Pharmacol Exp Ther 273: 445-454.

White SR, Harris GC, Imel KM, Wheaton MJ (1995b). Inhibitory effects of dopamine and methylenedioxymethamphetamine (MDMA) on glutamate-evoked firing of nucleus accumbens and caudate/putamen cells are enhanced following cocaine self-administration. Brain Res 681: 167-176.

Wise RA (2004). Dopamine, learning, and motivation. Nat Rev Neurosci 5: 483-494.

Xi Z-X, Ramamoorthy S, Shen H, Lake R, Samuvel DJ, Kalivas PW (2003). GABA transmission in the nucleus accumbens is alered after withdrawal from repeated cocaine. J Neurosci 23: 3498-3505.

Zahm DS (2000). An integrative neuroanatomical perspective on some subcortical substrates of adaptive responding with emphasis on the nucleus accumbens. Neurosci Biobehav Rev 24: 85-105.

Zhang X-F, Cooper DC, White FJ (2002). Repeated cocaine treatment decreases whole-cell calcium current in rat nucleus accumbens neurons. J Pharmacol Exp Ther 301: 1119-1125.

Zhang X-F, Hu X-T, White FJ (1998). Whole-cell plasticity in cocaine withdrawal: reduced sodium currents in nucleus accumbens neurons. J Neurosci 18: 488-498.

Supplementary Information accompanies the paper on the Neuropsychopharmacology website (http://www.nature.com/ npp) 